\title{
Investigation of Low-Dissipation Monotonicity-Preserving Scheme for Direct Numerical Simulation of Compressible Turbulent Flows
}

\author{
Jian Fang ${ }^{\mathrm{a}}$, Yufeng Yao ${ }^{\mathrm{b}}$, Zhaorui $\mathrm{Li}^{\mathrm{c}}$, Lipeng Lu ${ }^{\mathrm{a} *}$ \\ ${ }^{\text {a }}$ National Key Laboratory of Science and Technology on Aero-Engine Aero-Thermodynamics, School of \\ Energy and Power Engineering,, Beihang University, 37 Xueyuan Road, Beijing 100191, China \\ ${ }^{\mathrm{b}}$ Department of Engineering Design and Mathematics, University of the West of England, Bristol BS16 1QY, \\ UK \\ ${ }^{\mathrm{c}}$ Computational Physics and Methods, Los Alamos National Laboratory, P.O. Box 1663, Los Alamos, NM \\ 87545, USA \\ ${ }^{*}$ Corresponding author. Tel.: +861082317413, E-mail address: lulp@buaa.edu.cn (L. Lu) \\ Email address: fangjian19@gmail.com (J. Fang) \\ Email address: yufeng.yao@uwe.ac.uk (Y. Yao) \\ Email address: zli@lanl.gov (Z. Li)
}

\begin{abstract}
:
The influence of numerical dissipation on direct numerical simulation (DNS) of decaying isotropic turbulence at initial $\operatorname{Re}_{\lambda}=72$ and turbulent channel flow at $\operatorname{Re}_{\tau}=180$ is investigated respectively by using the seventh-order low-dissipation monotonicity-preserving (MP7-LD) scheme with different levels of bandwidth dissipation. It is found that for both benchmark test cases, small-scale turbulence fluctuations can be largely suppressed by high level of scheme dissipation, while numerical errors in terms of high-frequency oscillations
\end{abstract}


appear and could destabilize the computation if the dissipation is reduced to a very low level. Numerical studies show that reducing the bandwidth dissipation to $70 \%$ of the conventional seventh-order upwind scheme can maximize the efficiency of the MP7-LD scheme in resolving small-scale turbulent fluctuations and, in the meantime preventing the accumulation of non-physical numerical errors. By using the optimized MP7-LD scheme, DNS of an impinging oblique shock-wave interacting with a spatially-developing turbulent boundary layer is conducted at the incoming free-stream Mach number of 2.25 and shock angle of $33.2^{\circ}$. Simulation results of mean velocity profiles, wall surface pressure, skin friction and Reynolds stresses are validated against available experimental data and other DNS predictions in both the undisturbed equilibrium turbulent boundary layer region and the interaction zone, and good agreements are achieved. The turbulent kinetic energy transport equation is also analyzed and the balance of the equation is well preserved in the interaction region. This study demonstrates the capability of the optimized MP7-LD scheme for DNS of complex flow problems of wall-bounded turbulent flow interacting with shock-waves.

Keywords: Numerical dissipation, Monotonicity-preserving scheme, Direct numerical simulation, Shockwave/turbulent-boundary-layer interaction.

\section{Introduction}

Direct numerical simulation (DNS) and large-eddy simulation (LES) of compressible turbulent flows interacting with shock-waves raise challenges to numerical schemes, which are required to capture shockwaves without introducing spurious numerical oscillations and simultaneously resolve small-scale turbulent 
structures without evident dissipation. Even for weakly compressible turbulence, a certain level of numerical dissipation is needed in order to stabilize simulations by suppressing the aliasing errors resulting from the discretization of nonlinear convection terms [1]. Although some existing low- or non-dissipative methods, such as high-order central scheme with entropy splitting [2] or skew-symmetric splitting [3] can stabilize the computation by reducing the aliasing error without introducing ad hoc numerical dissipation, these methods cannot be effectively applied in near shock-wave regions, primarily due to the calculation of convection fluxes in non-conservative forms. Therefore, these methods often need to be used in conjunction with conservative schemes in the region near shock-waves [4, 5], which will cause further problems at the interfaces between different types of schemes. [6, 7] It is widely accepted that conventional shock-capturing schemes (such as essentially non-oscillatory (ENO) schemes [8, 9] and weighted essentially non-oscillatory (WENO) schemes [10]) are too dissipative to effectively resolve small-scale turbulence [11, 12]. Therefore, comparing with high-order central schemes, a denser grid must be used for conventional shock-capturing schemes aforementioned, and this will largely increase the computational cost of DNS or LES approach. For example, the effective bandwidth of the seventh-order WENO scheme is merely about $30 \%$ of the maximum bandwidth, i.e. $30 \% k_{\max }$ [11]; hence to achieve the same resolution with those high-order central schemes with the effective bandwidth of almost $100 \% k_{\max }$, three times more grid points are required in each direction, resulting in at least a factor of 27 in terms of grid points, memory and computational cost with possible further increase in computational time cost due to the decrease of time step. Therefore, lots of researchers tried to develop better shock-capturing schemes, including improved WENO schemes [13-17], high-order limiters [18-21] and filters [22, 23]. Among these shock-capturing schemes, the high-order monotonicity- 
preserving (MP) scheme proposed by Suresh and Huynh [21] has a good performance in preserving both the accuracy in smooth regions and the monotonicity near the discontinuities. Li and Jaberi [24] confirmed the good performance of the MP schemes in DNS of compressible turbulence with and without shocks and Jammalamadaka et al. [25, 26] adopted the seventh-order MP scheme in a series of DNS and LES of shockwave/turbulent-boundary-layer interactions (SWTBLI), which proved the capability of MP scheme in DNS and LES of SWTBLI. Some efforts tried to improve the performance of the MP scheme. Li et al. [27] proposed a sixth-order optimized MP scheme (OMP6), in which the dispersion errors are minimized . Their research demonstrated the better performance of the OMP6 scheme against the MP scheme in capturing small-scale flow structures. Fang et al. [28] recently proposed a low-dissipation MP (MP-LD) scheme, and they got an improvement of the effective bandwidth from $30 \% k_{\max }$ to almost $100 \% k_{\max }$ in DNS of isotropic turbulence by reducing 70\% of the bandwidth dissipation of the seventh-order MP scheme, which indicated the strong connection of the performance of upwind schemes to numerical dissipation. Following the studies of Li and Jaberi [24], Li et al. [27] and Fang et al. [28], the present paper further investigates the influences of the bandwidth dissipation of the MP7-LD scheme on DNS of various turbulent flow configurations. Firstly, the eigenvalue and the bandwidth property of the linear part of the MP7-LD scheme will be analyzed. Then, the effects of bandwidth dissipation will be extracted and investigated in weakly compressible isotropic turbulence and wall-bounded turbulence without the interference with the nonlinear errors or the order of accuracy, and the optimized value of the bandwidth dissipation will be proposed. Finally, DNS of impinging oblique shock-wave/turbulent-boundary-layer interactions at the free-stream Mach number 2.25 will be conducted by using the optimized MP7-LD scheme, and the results will be validated by comparing with the 
published experimental measurements and other DNS data, in order to demonstrate the applicability of the optimized MP7-LD scheme in DNS of complex wall-bounded turbulent flows with shock-waves.

The paper is organized as follows. In Section 2, we describe the governing equations, the numerical scheme and the properties of the MP7-LD scheme. The performances of the MP7-LD scheme are assessed and the influences of the bandwidth dissipation on DNS of isotropic turbulence and turbulent channel flow are studied in Section 3. In Section 4, we report the DNS of impinging shock-wave/turbulent-boundary-layer interaction by applying the optimized MP7-LD scheme together with results validation, comparison and discussions. Finally, in Section 5 we draw some conclusions.

\section{Governing Equation and Numerical Method}

\subsection{Governing Equations}

Three-dimensional unsteady compressible Navier-Stokes (N-S) equations in a general, time-invariant, curvilinear coordinate system are numerically solved for all turbulent flow cases considered hereafter. The NS equations are nondimensionalized with the reference length $L_{0}$, velocity $u_{0}$, temperature $T_{0}$, density $\rho_{0}$, and dynamic viscosity $\mu_{0}$. The resulting dimensionless parameters are Reynolds number $\operatorname{Re}=u_{0} L_{0} / \mu_{0}$ and Mach number $\mathrm{M}=u_{0} / \sqrt{\gamma R T_{0}}$. A constant Prandtl number $\operatorname{Pr}=\mu C p / h=0.72$ is used, where $C p=$ $\gamma R(\gamma-1)$ is the specific heat capacity of gas at a constant pressure and $h$ is the thermal conductivity. $R$ and $\gamma$ are the specific gas constant and the specific heat capacity ratio, which are both set to be constants as,

$R=287.1 \mathrm{~J} /(\mathrm{Kg} \cdot \mathrm{K})$ and $\gamma=1.4$.

This set of equations are written in the strong conservation form as, 


$$
\frac{\partial(J \boldsymbol{Q})}{\partial t}+\frac{\partial\left(\widehat{\boldsymbol{E}}-\widehat{\boldsymbol{E}}_{v}\right)}{\partial \xi}+\frac{\partial\left(\widehat{\boldsymbol{F}}-\widehat{\boldsymbol{E}}_{v}\right)}{\partial \eta}+\frac{\partial\left(\widehat{\boldsymbol{G}}-\widehat{\boldsymbol{G}}_{v}\right)}{\partial \zeta}=J \boldsymbol{S},
$$

where the coordinate transformation between the physical domain $(x, y, z)$ and the computational domain

$(\xi, \eta, \zeta)$, can be described by following equations as,

$$
x=x(\xi, \eta, \zeta), \quad y=y(\xi, \eta, \zeta), \quad z=z(\xi, \eta, \zeta)
$$

In Eq. (1), $J=|\partial(x, y, z) / \partial(\xi, \eta, \zeta)|$ is the Jacobian of the coordinate transformation and

$\boldsymbol{Q}=[\rho, \rho u, \rho v, \rho w, E]^{T}$ is the solution vector. The primary variables are the density $\rho$, the velocity

component $u, v, w$, and the total energy $E$. The temperature $T$ and pressure $P$ are related to the density $\rho$ via

the ideal gas law $P=\rho T /\left(\gamma M^{2}\right)$.

The convection and diffusion flux vectors in Eq. (1) are defined as,

$$
\widehat{\boldsymbol{E}}=\left[\begin{array}{c}
\rho \widehat{U} \\
\rho u \widehat{U}+\hat{\xi}_{x} P \\
\rho v \widehat{U}+\hat{\xi}_{y} P \\
\rho w \widehat{U}+\hat{\xi}_{z} P \\
(E+P) \widehat{U}
\end{array}\right], \quad \widehat{\boldsymbol{F}}=\left[\begin{array}{c}
\rho \widehat{V} \\
\rho u \widehat{V}+\hat{\eta}_{x} P \\
\rho v \widehat{V}+\hat{\eta}_{y} P \\
\rho w \widehat{V}+\hat{\eta}_{z} P \\
(E+P) \widehat{V}
\end{array}\right], \quad \widehat{\boldsymbol{G}}=\left[\begin{array}{c}
\rho \widehat{W} \\
\rho u \widehat{W}+\hat{\zeta}_{x} P \\
\rho v \widehat{W}+\hat{\zeta}_{y} P \\
\rho w \widehat{W}+\hat{\zeta}_{z} P \\
(E+P) \widehat{W}
\end{array}\right]
$$

and

$$
\widehat{\boldsymbol{E}}_{\boldsymbol{v}}=\left[\begin{array}{c}
0 \\
\hat{\xi}_{x i} \tau_{i 1} \\
\hat{\xi}_{x i} \tau_{i 2} \\
\hat{\xi}_{x i} \tau_{i 3} \\
\hat{\xi}_{x i} b_{i}
\end{array}\right], \quad \widehat{\boldsymbol{F}}_{\boldsymbol{v}}=\left[\begin{array}{c}
0 \\
\hat{\eta}_{x i} \tau_{i 1} \\
\hat{\eta}_{x i} \tau_{i 2} \\
\hat{\eta}_{x i} \tau_{i 3} \\
\hat{\eta}_{x i} b_{i}
\end{array}\right], \quad \widehat{\boldsymbol{G}}_{\boldsymbol{v}}=\left[\begin{array}{c}
0 \\
\hat{\zeta}_{x i} \tau_{i 1} \\
\hat{\zeta}_{x i} \tau_{i 2} \\
\hat{\zeta}_{x i} \tau_{i 3} \\
\hat{\zeta}_{x i} b_{i}
\end{array}\right]
$$

For the convenience of representing matrices and vectors, $\left(x_{1}, x_{2}, x_{3}\right),\left(u_{1}, u_{2}, u_{3}\right)$ and $\left(\xi_{1}, \xi_{2}, \xi_{3}\right)$ are used to be equivalent to $(x, y, z),(u, v, w)$ and $(\xi, \eta, \zeta)$, respectively, and the standard Einstein summation notation is used. In Eq. (2) and (3), the grid transformation $\left[\begin{array}{l}\hat{\xi}_{x i} \\ \hat{\eta}_{x i} \\ \hat{\zeta}_{x i}\end{array}\right]=J\left[\begin{array}{l}\partial \xi / \partial x_{i} \\ \partial \eta / \partial x_{i} \\ \partial \zeta / \partial x_{i}\end{array}\right]$ is used for all metric coefficients, and the contravariant velocity components and the total energy are written as, 


$$
\widehat{U}=\hat{\xi}_{x i} u_{i}, \quad \widehat{V}=\hat{\eta}_{x i} u_{i}, \quad \widehat{W}=\hat{\zeta}_{x i} u_{i},
$$

and

$$
E=\frac{1}{2}\left(\rho u_{i} u_{i}\right)+\frac{\rho T}{\gamma(\gamma-1) M^{2}}
$$

The stress tensor and the heat flux vector are expressed as,

$$
\tau_{i j}=\frac{\mu}{\operatorname{Re}}\left(\frac{\partial u_{i}}{\partial \xi_{k}} \frac{\partial \xi_{k}}{\partial x_{j}}+\frac{\partial u_{j}}{\partial \xi_{k}} \frac{\partial \xi_{k}}{\partial x_{i}}-\frac{2}{3} \delta_{i j} \frac{\partial u_{l}}{\partial \xi_{k}} \frac{\partial \xi_{k}}{\partial x_{l}}\right)
$$

and

$$
b_{i}=u_{j} \tau_{i j}+\frac{\mu}{\operatorname{PrRe}(\gamma-1) M^{2}} \frac{\partial T}{\partial \xi_{k}} \frac{\partial \xi_{k}}{\partial x_{i}}
$$

The dynamic viscous coefficient $\mu$ is calculated via the Sutherland law as,

$$
\mu=T^{1.5} \frac{T_{S} / T_{0}+1}{T+T_{S} / T_{0}}
$$

where $T_{0}=216.65 \mathrm{~K}$ and $T_{S}=110.4 \mathrm{~K}$ are used in all simulations.

The source term $\boldsymbol{S}$ in Eq. (1), unless specific defined, is assumed to be 0 . Unless otherwise described, all the variables in the present paper are in the dimensionless form.

\subsection{Numerical Scheme}

We take the derivative of a general function $F(x)$ on a set of one-dimensional uniformly distributed grid points $\left(x_{i}=i \Delta, i=0,1, \cdots, N\right)$ with the grid spacing $\Delta$ to illustrate the numerical scheme. Thus, the derivative $\partial F(x) / \partial x$ at $x_{i}$ can be approximated as,

$$
\frac{\partial F(x)}{\partial x}=\frac{\left(\hat{F}_{i+1 / 2}-\hat{F}_{i-1 / 2}\right)}{\Delta}+O\left(\Delta^{m}\right)
$$


where $m$ stands for the order of the accuracy of the scheme and $\widehat{F}_{i+1 / 2}$ in Eq. (10) is the numerical flux function at an interface location of $x_{i+1 / 2}=\left(x_{i}+x_{i+1}\right) / 2$, which can be reconstructed based on a specific numerical scheme using the values at several neighboring grid points.

Considering the MP7-LD scheme [28], its linear part is given as,

$$
\widehat{F}_{i+1 / 2}=\frac{1}{280}\left(a_{-3} F_{i-3}+a_{-2} F_{i-2}+a_{-1} F_{i-1}+a_{0} F_{i}+a_{1} F_{i+1}+a_{2} F_{i+2}+a_{3} F_{i+3}-b_{7} F_{i+4}\right),
$$

in which $b_{7}$ is a free parameter to determine the relative contribution of the point $i+4$. Substituting Eq. (11)

into Eq. (10), the first-order derivative can be approximated as,

$$
\frac{\partial F(x)}{\partial x} \approx \sum_{n=-4}^{4} \alpha_{n} F_{i+n},
$$

where the finite difference coefficients $\alpha_{n}$ are calculated as,

$$
\left[\begin{array}{c}
\alpha_{-4} \\
\alpha_{-3} \\
\alpha_{-2} \\
\alpha_{-1} \\
\alpha_{0} \\
\alpha_{1} \\
\alpha_{2} \\
\alpha_{3} \\
\alpha_{4}
\end{array}\right]=\left[\begin{array}{c}
-a_{-3} \\
a_{-3}-a_{-2} \\
a_{-2}-a_{-1} \\
a_{-1}-a_{0} \\
a_{0}-a_{1} \\
a_{1}-a_{2} \\
a_{2}-a_{3} \\
a_{3}+b_{7} \\
-b_{7}
\end{array}\right] .
$$

By employing the Taylor series expansion and keeping the seventh-order accuracy, the values of $a_{-3}, a_{-2}, a_{-1}, a_{0}, a_{1}, a_{2}, a_{3}$ are determined in terms of the free parameter $b_{7}$, as given below,

$$
\left[\begin{array}{l}
a_{-3} \\
a_{-2} \\
a_{-1} \\
a_{0} \\
a_{1} \\
a_{2} \\
a_{3}
\end{array}\right]=\left[b_{7}-2,-7 b_{7}+\frac{50}{3}, 21 b_{7}-\frac{202}{3},-35 b_{7}+\frac{638}{3}, 35 b_{7}+\frac{428}{3},-21 b_{7}-\frac{76}{3}, 7 b_{7}+\frac{8}{3}\right]^{T} .
$$


For $b_{7}=0$, Eq. (12) is recovered to the conventional seventh-order upwind scheme. On the contrary, when $b_{7}$ is set to be 1, the Eq. (12) becomes the explicit eighth-order central scheme, which is a nondissipative scheme.

\subsubsection{Eigenvalue Analysis}

Consider the one-dimensional advection equation,

$$
\frac{\partial \phi(x, t)}{\partial t}+\frac{\partial \phi(x, t)}{\partial x}=0
$$

with the solution vector of $\boldsymbol{\phi}=\left(\phi_{1}, \phi_{2}, \cdots, \phi_{N}\right)$ on a set of $N$ discrete grid points. Its semi-discrete form can

be written as,

$$
\frac{d \boldsymbol{\phi}}{d t}=\frac{1}{\Delta} \boldsymbol{B} \boldsymbol{\phi},
$$

where $\boldsymbol{B}$ is the coefficient matrix of the scheme in Eq. (12).

The eigenvalue analysis states that the scheme will be stable if all eigenvalues of the matrix $\boldsymbol{B}$ have nonpositive real parts and also the time step falls within the stability region of the time-integration scheme. The eigenvalue spectra of $\boldsymbol{B}$ for $N=200$ with the periodic boundary condition are given in Figure 1. It can be seen that the eigenvalue has non-positive real parts only when $b_{7} \leq 1$. Taking the conventional seventh-order upwind scheme $\left(b_{7}=0\right)$ as the baseline scheme, and increasing parameter $b_{7}$ from 0 to 1 , the scheme of Eq. (12) is becoming more stable as the eigenvalues are closer to the origin and therefore allowing a larger time step. On the contrary, reducing $b_{7}$ from 0 , the scheme is still stable, but requires a smaller time step. The scheme with $b_{7}>1$ is unstable and therefore should be avoid. 


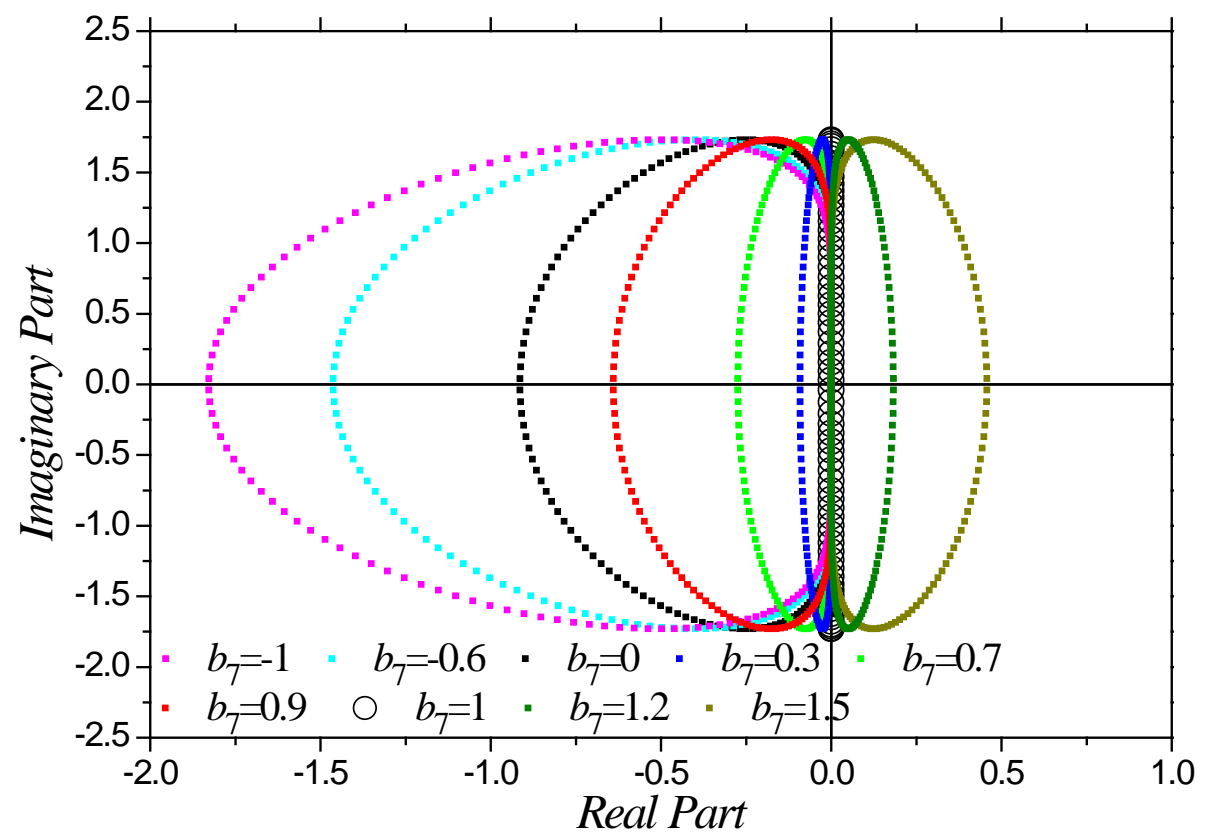

Figure 1. The eigenvalue spectra of matrix $B$ for $N=200$ points and the periodic boundary condition.

\subsubsection{Fourier Analysis}

By using the Fourier analysis, the bandwidth properties of Eq. (12) with different values of $b_{7}$ from -1 to 1 are presented in Figure 2, in which the relation of the modified wavenumber $\hat{k}$ and the wavenumber $k$ is given as,

$$
\hat{k}=-i \sum_{n=-4}^{4} \alpha_{n} e^{i k n}
$$

As shown in Figure 2(a), the real part of the modified wavenumber that represents the bandwidth

resolution remains unchanged while varying parameter $b_{7}$ from -1 to 1 (i.e. the results are collapsed).

However, the numerical dissipation indicated by the negative value of the imaginary part of the modified wavenumber in Figure 2(b) is consistently reduced to zero by increasing the parameter $b_{7}$ from 0 to 1 . In contrary, reducing $b_{7}$ from 0 to -1 , the bandwidth dissipation of Eq. (12) will be increased. 

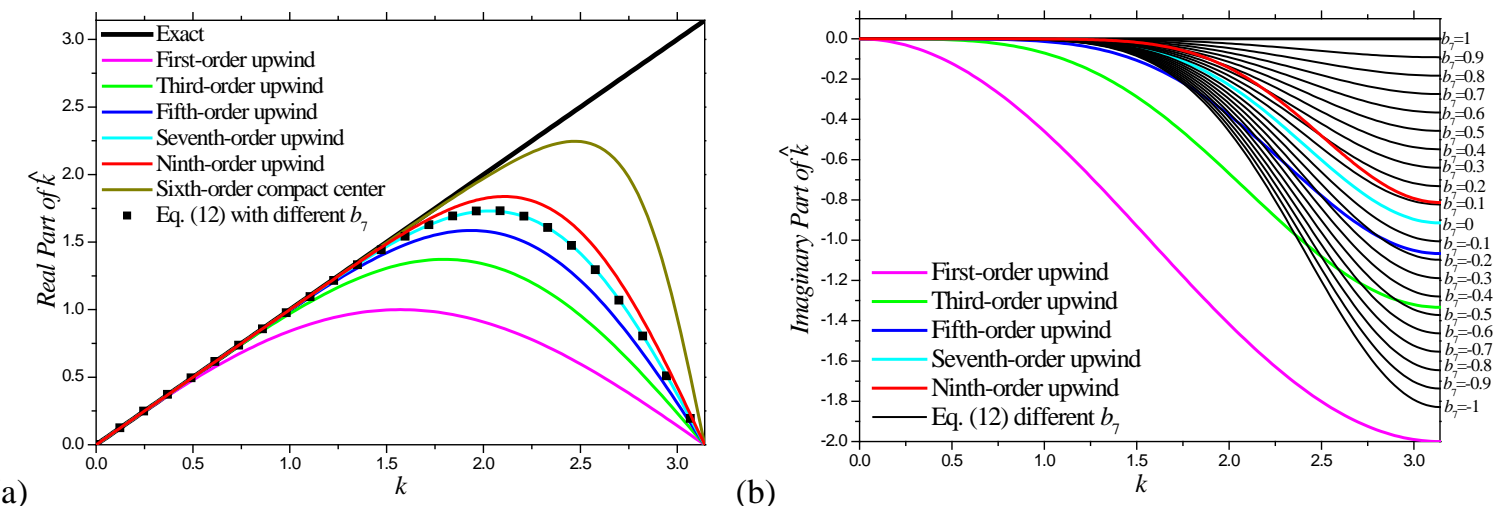

(b)

Figure 2. Bandwidth properties of the linear part of the MP7-LD scheme. (a) real part of the modified wavenumber; (b) imaginary part of the modified wavenumber.

By integrating the imaginary part of the modified wavenumber, the integral bandwidth dissipation can be defined as,

$$
\Sigma=\int_{0}^{\pi}-\operatorname{Im}(\hat{k}) d k
$$

in which $\operatorname{Im}(\quad$ ) means the imaginary part of a complex variable.

The relation of the integral bandwidth dissipation $\Sigma$ in Eq. (18) with respect to parameter $b_{7}$ is illustrated in Figure 3, from which the linear relation of the integral bandwidth dissipation and parameter $b_{7}$ can be seen.

For example, by setting $b_{7}=0.2$, the integral dissipation will be reduced to that of the conventional ninthorder upwind scheme. For $b_{7}=-1$, the integral dissipation is close to that of the conventional third-order upwind scheme and the integral dissipation of the conventional fifth-order upwind scheme can be achieved by setting $b_{7}=-0.332$. The linear relation between the bandwidth dissipation and parameter $b_{7}$ suggests that with the increased value of parameter $b_{7}$ from 0 to 1 , a percentage of bandwidth dissipation $b_{7} \times 100 \%$ of its original level will be reduced; therefore, the numerical dissipation of the MP7-LD scheme can be conveniently tuned to a required level. 


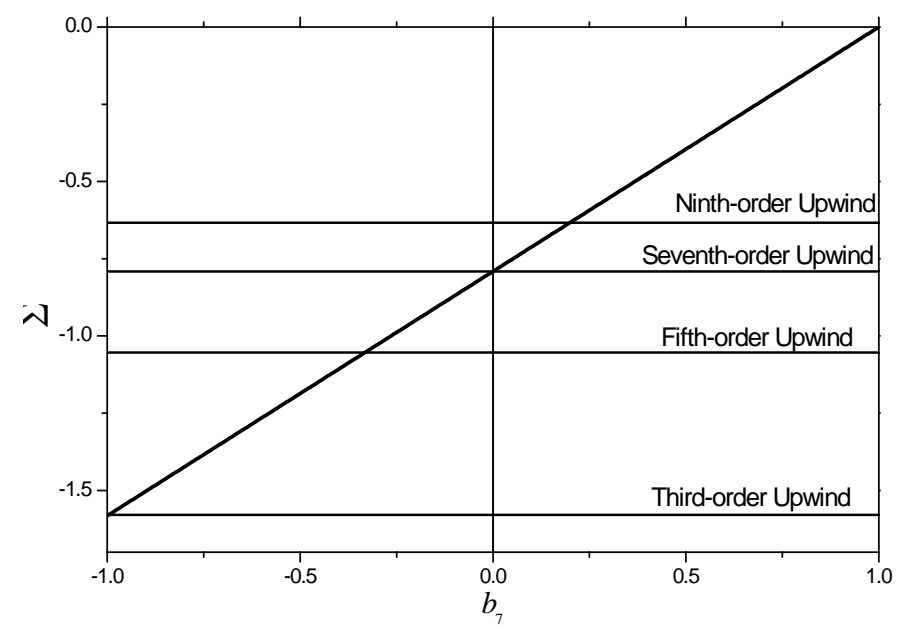

Figure 3. Integral Bandwidth Dissipation with the parameter $b_{7}$.

In order to resolve small-scale turbulent structures and also to preserve turbulence energy, the numerical dissipation should be kept as low as possible. In other word, parameter $b_{7}$ should be close to 1 . However, in practical simulations, a certain level of numerical dissipation is necessary to suppress the aliasing errors [29]. Therefore, parameter $b_{7}$ is suggested to be set between 0 and 1 , in consideration of both the efficiency and the stability of the computation.

In the MP7-LD scheme, a shock sensor proposed by Ducros et al. [30] is adopted to replace the smoothness criterion of the original MP scheme, and this shock sensor at a grid point $i$ can be expressed as,

$$
\Omega_{i}=\left|\frac{P_{i+1}-2 P_{i}+P_{i-1}}{P_{i+1}+2 P_{i}+P_{i-1}}\right| \frac{\left(\partial u_{k} / \partial x_{k}\right)^{2}}{\left(\partial u_{k} / \partial x_{k}\right)^{2}+\omega_{k} \omega_{k}+\varepsilon}
$$

where $P_{i}$ is static pressure at $i, \omega_{k}=\partial u_{j} / \partial x_{i}-\partial u_{i} / \partial x_{j}$ is the vorticity component and a small value $\varepsilon=10^{-30}$ is used to avoid zero dividing. This shock sensor is proven to be the best one among four different shock sensors assessed by Pirozzoli [1] recently. A threshold value $\sigma=0.01$ is used to active the MP limiter, which is the same value suggested by Lo et al. [31]. For one-dimensional problems, due to the inexistence of 
the vorticity, the smoothness criterion of the fifth-order WENO-JS scheme [32] is used as a shock sensor,

which is given as,

$$
\Omega_{i}=\left[\frac{1}{4}\left(P_{i+1}-P_{i-1}\right)^{2}+\frac{13}{12}\left(P_{i+1}-2 P_{i}+P_{i-1}\right)^{2}\right]^{2} .
$$

The application of the MP7-LD scheme is summarized in following steps:-

1. The original convection fluxes values at the interface are calculated by using the bandwidth dissipation optimized upwind scheme, i.e. Eq. (11) with a selected optimized parameter $b_{7}$.

2. The shock sensor $\Omega_{i}$ is calculated from Eq. (19) or (20) depending on the dimensionality of the problem.

3. If $\Omega_{i}>\sigma$, the MP limiter described in reference [21] will be incorporated to determine the final interface value to preserve the monotonicity.

4. Otherwise, the final interface values will be determined as the original interface value.

5. The derivative is finally calculated with the neighboring interface values via Eq. (10).

For the hyperbolic system of the Navier-Stokes equations, upwind schemes in the present paper are used together with the Steger-Warming flux vector splitting method [33]. To reduce numerical errors, the conventional fluxes are evaluated in the local characteristic space [34] with a pair of Roe-averaged eigenvectors of the Jacobian matrix to transform the convection fluxes between the physical space and the characteristic space [35]. 
A sixth-order compact central scheme (COMP6) [36], which has been widely adopted as a high-order method in DNS and LES of weakly compressible turbulence, is used as a reference scheme in the present study. This scheme is expressed as,

$$
\frac{1}{3} F_{i-1}^{\prime}+F_{i}^{\prime}+\frac{1}{3} F_{j+1}^{\prime}=\left(F_{i+2}-F_{i-2}\right) / 36+7\left(F_{i+1}-F_{i-1}\right) / 9
$$

A tenth-order compact filter is incorporated as a stabilizer when the COMP6 scheme is used to solve convection terms, whose expression is,

$$
\alpha_{f} \hat{F}_{i-1}+\widehat{F}_{i}+\alpha_{f} \hat{F}_{i+1}=\sum_{n=0}^{5} a_{n}\left(F_{i+n}+F_{i-n}\right) / 2
$$

where $\hat{F}$ is the filtered variable and $\alpha_{f} \in(-0.5,0.5)$ is the filter coefficient used to determine the strength of the filter and $a_{n}$ is calculated according to $\alpha_{f}$. The details of the filter and its property can be found in a paper by Visbal and Gaitonde [37]. In the present study, $\alpha_{f}=0.49$ is used for all the test cases, which restricts the filter only at very high wavenumbers.

The diffusion terms of N-S equations are solved with the COMP6 scheme of Eq. (21). The primitive variables $u_{i}$ and $T$ are firstly differentiated, and the stress tensor as well as the heat flux vector are then formed at each node. The diffusion terms are then computed by differentiating the first-order derivative values with another application of Eq. (21). This method is more efficient than the direct calculation of second-order derivatives, although the later method can be more numerically stable. After all the spatial terms are solved, the third-order TVD Runge-Kutta method is used for the time integration [38]. 


\section{Numerical Experiments}

\subsection{Shu-Osher Problem}

The problem of a moving Mach 3 shock-wave interacting with a sinusoidal density wave, known as the Shu-Osher problem [8] is firstly considered. The numerical dissipation is investigated by solving onedimensional Euler equations on the grids with 150 and 200 points. The density profiles at non-dimensional time unit $t=1.8$ from the MP7-LD scheme with parameter $b_{7}=0,0.3,0.7$ and 0.9 are compared with the gridconverged solution, which is calculated by the original MP7 scheme on a very fine grid with 10000 points (see Figure 4).

(a)

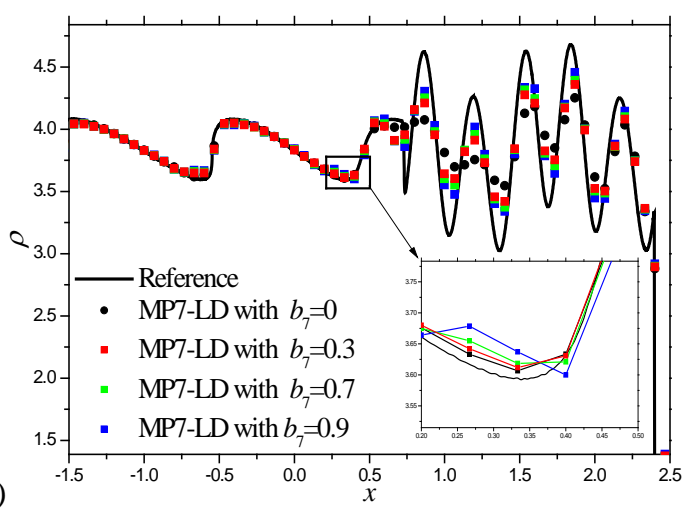

(b)

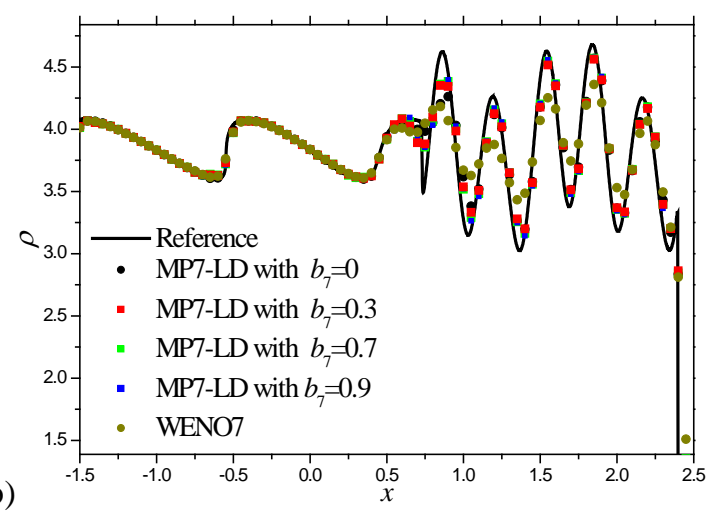

Figure 4. Comparison of density distribution at $t=1.8$ for the Shu-Osher problem on 150 points (a) and 200 points (b).

As indicated in Figure 4 (a), immediately left the normal shock-wave located at about $x=2.4$, the

monotonic increase of the density wave amplitude with the decrease of numerical dissipation can be realized.

Therefore, the result is gradually improved by increasing parameter $b_{7}$ from 0 to 0.7 . However, with further reducing the numerical dissipation by setting $b_{7}=0.9$, undesirable numerical oscillations appear at the compression wave (see zoomed figure inserted in Figure 4 a), and contaminate the result. Therefore, for this 
problem, parameter $b_{7}=0.7$ is the optimized option among the four values tested. However, when the number of the points increases from 150 to 200, the difference between the results from MP7-LD scheme with different values of $b_{7}$ is not obvious and they are all close to the reference. Comparing with the original WENO scheme of Jiang and Shu [10], the better performance of the MP7-LD scheme with any values of $b_{7}$ can be get.

\subsection{Homogeneous Isotropic Turbulence}

DNS of weakly compressible isotropic turbulence is carried out to further assess the influence of the numerical dissipation. Results of the MP7-LD scheme with parameter $b_{7}=0,0.3,0.7$ and 0.9 as well as the COMP6 scheme are analyzed and compared with the DNS data by using the pseudo-spectral method. The initial flow field is a divergence-free velocity field (seeded with random white noise) with uniform thermal variables, same as that of the study by Samtaney et al. [39]. The initial kinetic energy spectrum is given as,

$$
E(k)=A k^{4} e^{-2\left(k / k_{0}\right)^{2}},
$$

where $k$ is the wavenumber, $k_{0}=8$ is the wavenumber at which the spectrum peak locates, and $A=1.3 \times$ $10^{-4}$ is a constant to specify initial kinetic energy.

The root mean square (RMS) velocity fluctuation $u_{R M S}$, Taylor micro-scale $\lambda$ and the corresponding Reynolds number $\operatorname{Re}_{\lambda}$ are defined as,

$$
\begin{gathered}
u_{R M S}=\sqrt{\left\langle u_{k}{ }^{\prime \prime} u_{k}{ }^{\prime \prime}\right\rangle / 3}, \\
\lambda=\sqrt{\frac{\left\langle u_{k}^{\prime \prime} u_{k}^{\prime \prime}\right\rangle}{\left\langle\left(\partial u^{\prime \prime} / \partial x\right)^{2}\right\rangle+\left\langle\left(\partial v^{\prime \prime} / \partial y\right)^{2}\right\rangle+\left\langle\left(\partial w^{\prime \prime} / \partial z\right)^{2}\right\rangle}} \\
\operatorname{Re}_{\lambda}=\operatorname{Re} u_{R M S} \bar{\rho} \lambda /\langle\mu\rangle,
\end{gathered}
$$


where $\langle F\rangle$ denotes the Favré averaged value of $F$, which is calculated as, $\langle F\rangle=\overline{\rho F} / \bar{\rho}$. And “-” means statistical averaging, which equals to the volume averaging for this case. The fluctuation is expressed as $F^{\prime}=F-\bar{F}$ and $F^{\prime \prime}=F-\langle F\rangle$

The turbulent Mach number $M_{T}$, which is used to measure the compressibility of turbulence, is defined as,

$$
M_{T}=\sqrt{\left\langle u_{k}^{\prime \prime} u_{k}^{\prime \prime}\right\rangle} /\langle C\rangle,
$$

where $C=\sqrt{T} / M$ is the nondimensionalized speed of sound.

Initially, $M_{T}$ is set to be 0.3 , and $\operatorname{Re}_{\lambda}$ is set to be 72 . A total of $128^{3}$ grid points are used to discretize a cubic computational domain of $2 \pi^{3}$, with grid resolution close to the Kolmogorov length scale $\left(k_{\max } \eta_{0} \approx\right.$ 0.9 , with $k_{\max }=64$ and $\eta_{0}$ representing the initial Kolmogorov length scale) [39]. Due to the weak compressibility of the present case, the MP limiter is inactivated by the shock sensor; therefore, the results are only determined by the linear part of the MP7-LD scheme, i.e. Eq. (11) with different values of parameter $b_{7}$.

The temporal evolutions of the turbulent kinetic energy (TKE $\left.=\frac{1}{2}\left\langle u_{k}^{\prime \prime} u_{k}^{\prime \prime}\right\rangle\right)$ and enstrophy $\left(\Omega_{E N}=\right.$ $\left.\frac{1}{2}\left\langle\omega_{k} \omega_{k}\right\rangle\right)$ normalized with their initial values are compared in Figure 5, in which the time is normalized with the initial large-eddy turnover time $\tau_{0}$.
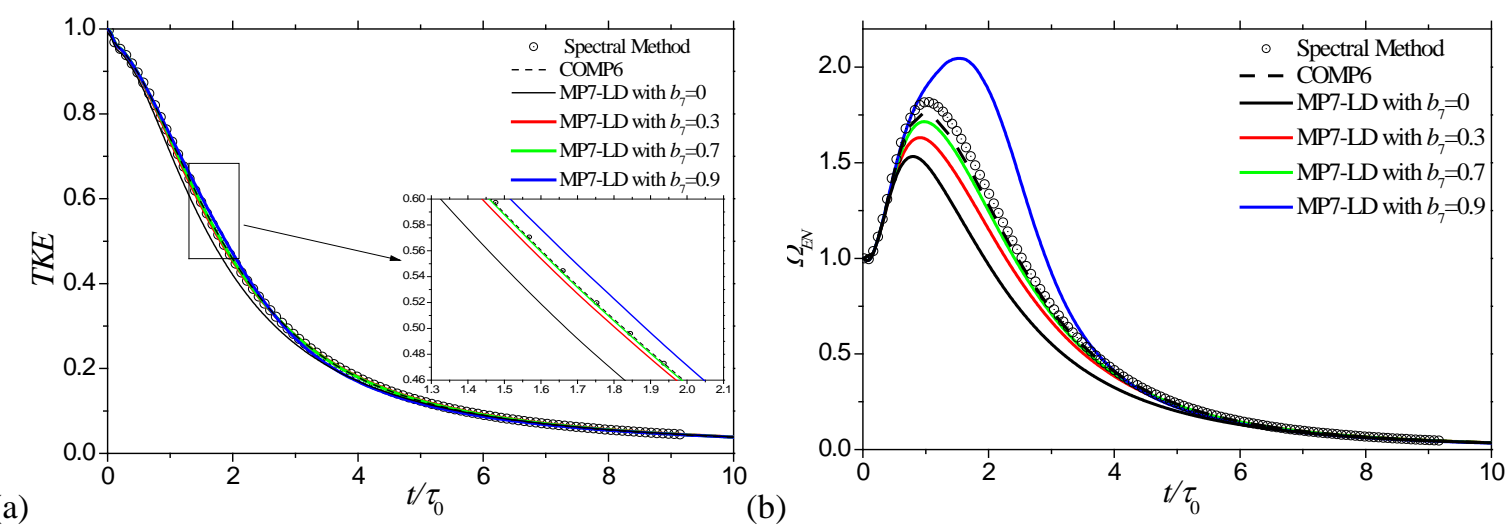

Figure 5. Temporal evolutions of turbulent kinetic energy (a) and enstrophy (b). 
It can be seen from Figure 5(a) that, the temporal evolutions of turbulent kinetic energy from all schemes tested agree reasonably well with each other, which indicates insensitive of the TKE prediction to the numerical dissipation. However, a more detailed comparison seen in a zoom sub-figure inserted in Figure 5(a) shows a faster decay of TKE for the MP7-LD scheme with $b_{7}=0$ and a slightly over-prediction of TKE for $b_{7}=0.9$ in comparison with the benchmark results of the spectral method and the COMP6 scheme. The possible reasons for this could be attributed to the suppression of turbulent fluctuations by the over-excessive numerical dissipation of small $b_{7}$ value and unsuppressed numerical oscillations for large $b_{7}$ value, respectively.

According to enstrophy variations in Figure 5(b), which is closely related to small-scale turbulence fluctuations [40], the differences among results from different parameter $b_{7}$ are more distinguishable. The enstrophy obtained by the MP7-LD scheme with $b_{7}=0$ has shown the lowest peak value and the fastest decay rate, which indicates the strongest suppression of small-scale turbulence fluctuations. While increasing $b_{7}$, i.e. reducing the bandwidth dissipation, the level of enstrophy is becoming higher. While $b_{7}$ is set to be 0.7 , the result of the MP7-LD scheme is very close to that of COMP6 scheme, which indicates the similar performances in terms of resolving turbulence fluctuations of these two schemes. However, when further reduce the numerical dissipation by setting $b_{7}$ to be 0.9 , the enstrophy is largely overpredicted, which could be attributed to the rise of small-scale unphysical numerical oscillations.

The energy spectrum $E(k)$ at an instantaneous time of $t=2 \tau_{0}$ (see Figure 6) also shows the unphysical rise of $E(k)$ at high wavenumber region for the MP7-LD scheme with $b_{7}=0.9$, which is consistent with the overprediction of the enstrophy observed in Figure 5(b). Therefore, it can be concluded that while the 
numerical dissipation is tuned too low (i.e. parameter $b_{7}$ is close to 1.0 ), the resulting unphysical small-scale fluctuations will contaminate the flow field significantly. With further accumulation of numerical errors at high wavenumber range, the computation would become unstable and most likely to be crashed eventually.

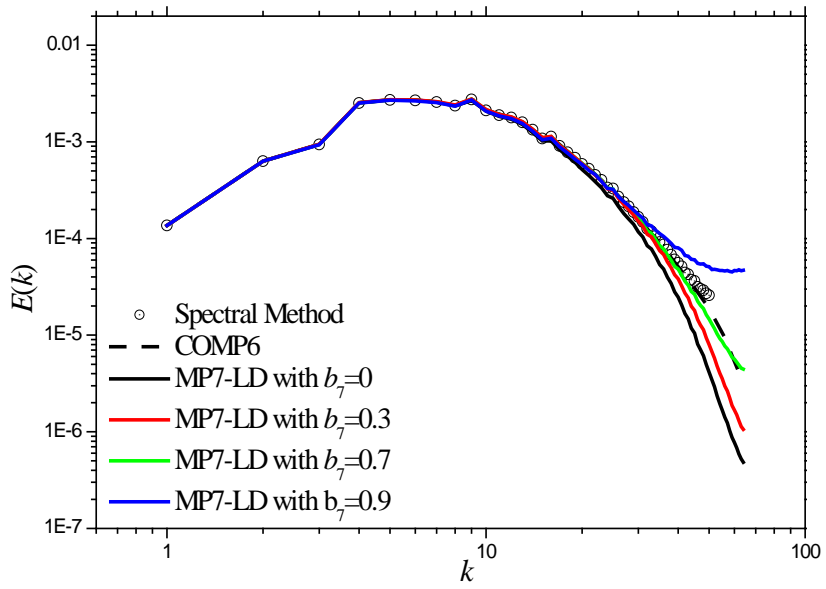

Figure 6. Instantaneous energy spectra at $t=2 \tau_{0}$.

For the MP7-LD scheme with parameter $b_{7}=0$, the turbulent energy at high wavenumbers is obviously underpredicted, which indicates the suppression of small-scale turbulence fluctuations by the high level of bandwidth dissipation at $b_{7}=0$. While increasing $b_{7}$ from 0 to 0.7 , an improved prediction of the energy spectrum at high wavenumbers can be achieved, and this confirms the critical impact of the numerical dissipation level on resolving small-scale turbulent motions. For $b_{7}=0.7$, the result of present MP7-LD scheme is very close to that of the COMP6 scheme and the spectral method.

By using the vortex identification scheme of the second invariant of the velocity gradient tensor, i.e. the Q criterion [41], the turbulent coherent structures from simulations using the MP7-LD scheme with $b_{7}=0.7$ and $b_{7}=0.9$ respectively at an instantaneous time of $t=2 \tau_{0}$ are visualized in Figure 7 . The classic randomly distributed worm-like vortices can be identified in Figure 7(a). However, small-scale oscillations (pointed by 
the red arrow) appear in Figure 7(b), which is a visual evidence of the small-scale numerical errors that will

appear when the numerical dissipation is tuned too low.

(a)

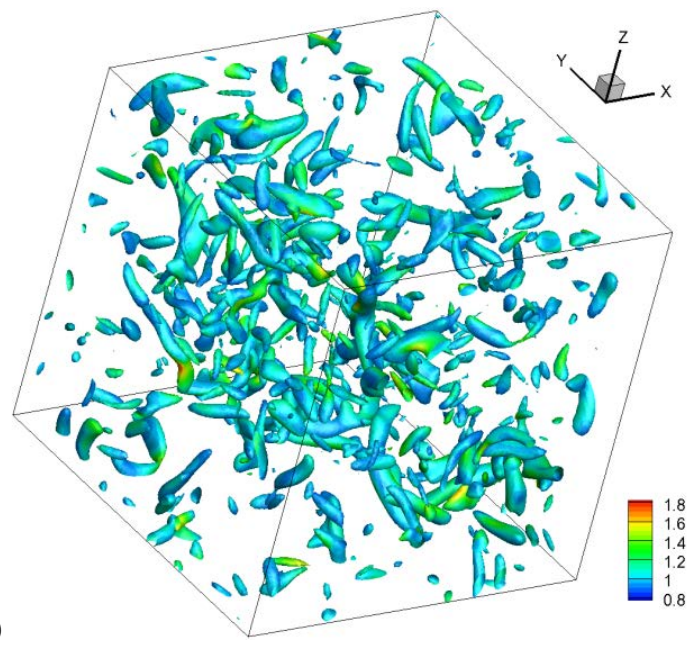

(b)

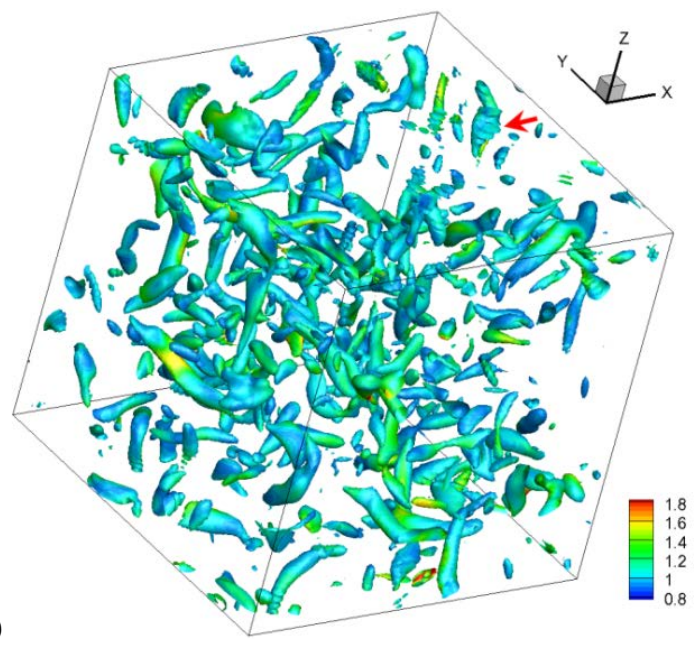

Figure 7. Turbulent coherent structures identified by $\mathbf{Q}$ criterion and colored by vorticity intensity.

The results are obtained using the MP7-LD scheme with (a): $b_{7}=0.7 ;(b): b_{7}=0.9$.

The computing efficiency of the MP7-LD and COMP6 scheme is also evaluated. Firstly, we compare the

CPU times cost for 300 times calculation of the convection fluxes for the two schemes, during which the

shock sensor Eq. (19) and tenth-order compact filter Eq. (22) are calculated every step for MP7-LD and

COMP6 respectively. Then, the CPU time consuming for 100 time steps advance in DNS of the present case

is given. The results are tabulate in Table 1 , from which we can get that, although the calculation of the

convection fluxes with the MP7-LD scheme costs almost 4 times CPU time against the COMP6 scheme, the

CPU time of solving the whole N-S equations is only increased less than 2 times.

Table 1 CPU time consuming

\begin{tabular}{ccc}
\hline \hline & $\begin{array}{c}\text { Calculating inviscid fluxes for 300 } \\
\text { times (second) }\end{array}$ & $\begin{array}{c}\text { Solving N-S equations for 100 } \\
\text { time steps (second) }\end{array}$ \\
\hline MP7-LD & 291.5 & 518.2 \\
\hline COMP6 & 74.2 & 278.2 \\
\hline \hline
\end{tabular}


In case of higher $M_{T}$ isotropic turbulence, shocklets will be generated, which is regarded a rigorous test case for the numerical scheme. Therefore, the forced isotropic turbulence at $M_{T}=0.8$ and $R e_{\lambda}=145$ is directly simulated to test the capability of the MP7-LD scheme in capturing shocklets. To maintain higher level of the turbulence kinetic energy, the divergence-free random forces at the largest scale are added to the momentum and the energy equations in the same way as Kida and Orszag [42]. The MP7-LD scheme with $b_{7}=0.7$ is used to solve the convection terms with a total of $256^{3}$ grid points. The instantaneous fields of flow variables are shown in Figure 8, in which the randomly generated shocklets can be identified as large density gradients and/or negative velocity divergences. According to Figure 8(c), the adopted shock sensor Eq. (19) and the selection of threshold can identify all the regions, where shocklets are possibly existent. The simulation demonstrates the capability of proposed the MP7-LD scheme in capturing shock-waves. 
(a)

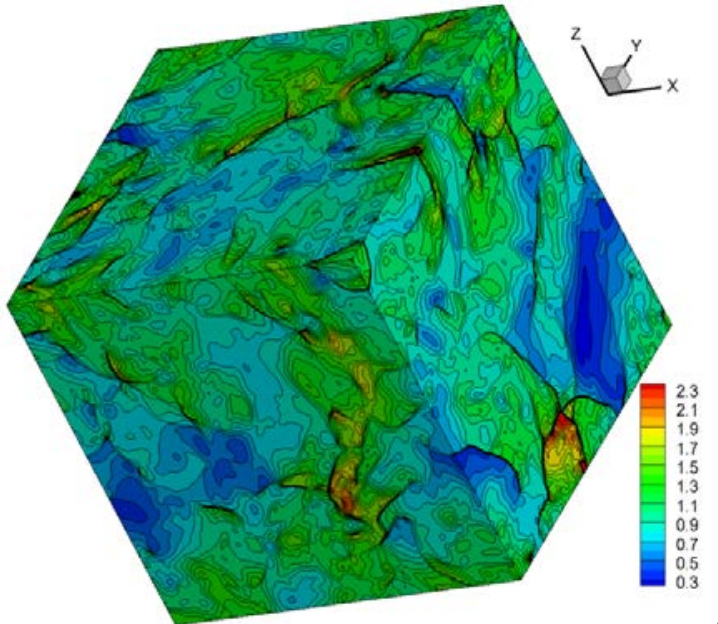

(b)
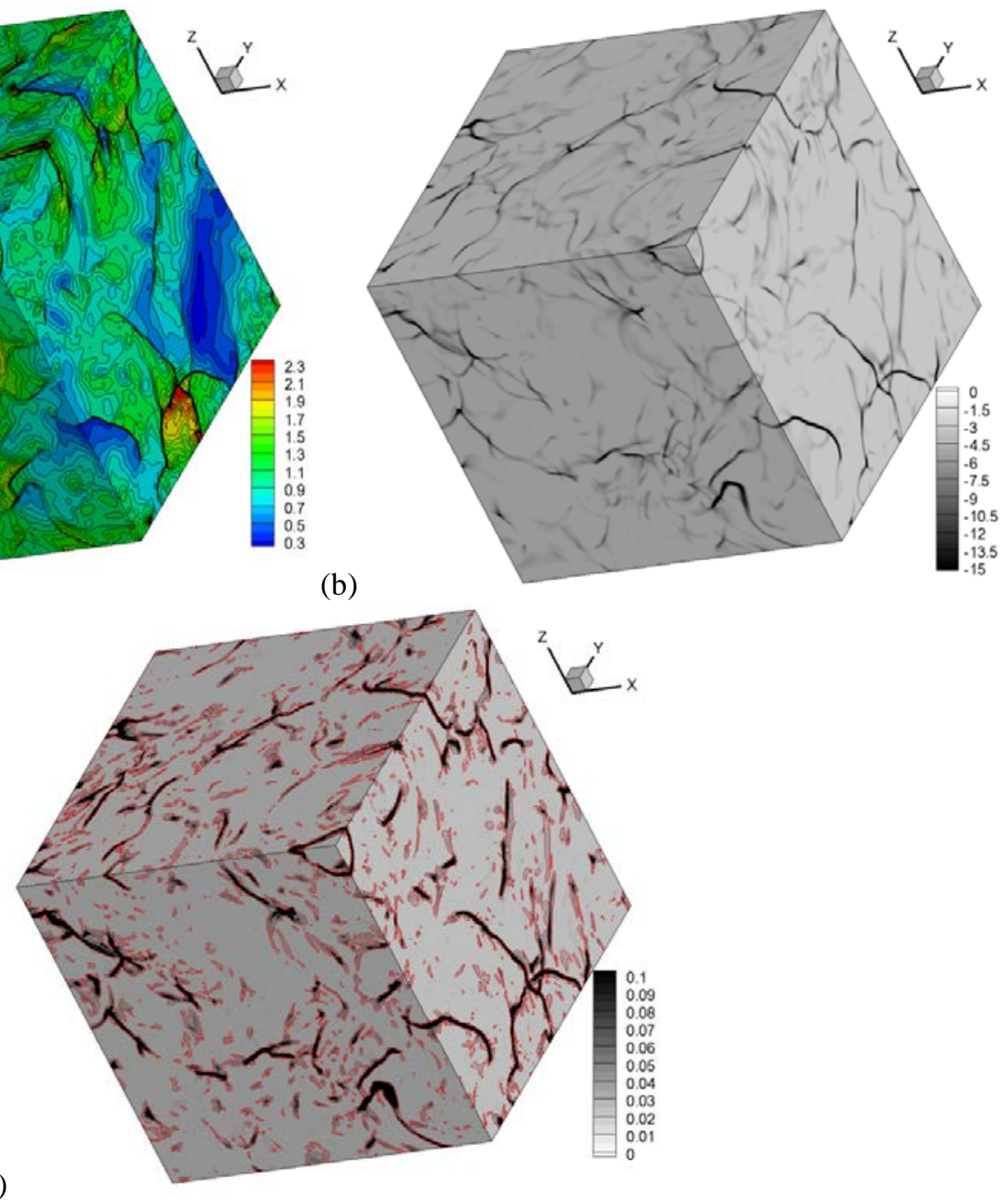

Figure 8. Instantaneous field of (a): density, (b): velocity divergence and (c): shock sensor. The threshold value $\sigma=0.01$ is marked with the red contour lines.

\subsection{Turbulent Channel Flow}

The influence of parameter $b_{7}$ is continued to be investigated in DNS of a channel flow configuration with two homogeneous directions in streamwise and the spanwise. The computation domain is $2 \pi \times 2 \times \pi$ in streamwise ( $x$ ), wall-normal $(y)$ and spanwise (z) direction respectively, with the reference length $L_{0}$ being the channel half-height $h$. The reference Reynolds number Re is 3000 based on $L_{0}$, bulk density $\rho_{0}$, bulk velocity $u_{0}$ and viscosity $\mu_{0}$ at wall Temperature $T_{0}$. The corresponding friction Reynolds number is $\operatorname{Re}_{\tau} \approx$ 
180, which is close to the incompressible spectral DNS of Moser et al. [43] The reference Mach number based on $u_{0}$ and $T_{0}$ is 0.5 , which defines a weakly compressible flow. [44]

The domain is discretized with $96 \times 96 \times 96$ grid points, which are uniformly distributed in $x$ and $z$ directions and hyperbolically clustered towards top and bottom walls in the $y$ direction. The grid spacings in the streamwise and the spanwise directions are $\Delta x^{+} \approx 12$ and $\Delta z^{+} \approx 6$ in wall units, respectively (based on the wall friction velocity $u_{\tau}=\sqrt{\left.\frac{1}{\operatorname{Re}} \frac{\mu \partial u / \partial y}{\rho}\right|_{W}}$ and the viscous length scale $l_{v}=\frac{1}{\operatorname{Re}} \frac{\mu_{W}}{\rho_{W} u_{\tau}}$ ). In the wall-normal direction, the first grid point away from the wall is $y_{1}^{+} \approx 0.7$ and the maximum grid spacing is 7.5 wall units at the centerline of the channel. The grid matches the resolution for DNS of wall-bounded turbulence recommended by Sagaut [45].

The flow is driven by a uniform streamwise body force, which is defined as the source term in the N-S equations as,

$$
\boldsymbol{S}=\left(\begin{array}{c}
0 \\
F_{x} \\
0 \\
0 \\
F_{x} u_{b}
\end{array}\right),
$$

in which, $F_{x}$ is the body force, $u_{b}=\frac{\overline{\rho u}_{x z}}{\bar{\rho}_{x z}}$ is the bulk velocity and $\bar{\rho}_{x z}$ means the density averaged in the $x$ and the $z$ directions. The body force is calculated every time step, therefore, it varies in time such that the total mass flux $\int_{0}^{h} \overline{\rho u}_{x z} d y$ remains constant during the simulation. The body force calculation follows that of Lenormand et al. [44], which is briefly described here as,

$$
F_{x}^{n+1}=F_{x}^{n}-\frac{\Delta t}{L_{y} L_{z}}\left[2\left(Q^{n+1}-Q_{0}\right)-0.2\left(Q^{n}-Q_{0}\right)\right]
$$


where $Q_{0}, Q^{n}$ and $Q^{n+1}$ are, respectively, the target mass flux supposed to be conserved, the mass flux at time step $n$ and the first-order prediction of the mass flux at time step $n+1$, given as,

$$
Q^{n+1}=Q^{n}+\Delta t\left(L_{y} L_{z} F_{x}^{n}-L_{z} \tau_{W}\right)
$$

with $\tau_{W}$ being the spatial averaged wall viscous stress.

The periodical boundary condition is applied in the $x$ and the $z$ directions, and isothermal nonslip condition is used at the top and bottom walls. The MP7-LD scheme with $b_{7}=0,0.7$ and 0.9 are assessed and results from the COMP6 scheme is used as the reference data. The statistics are got by averaging the flow field along the $x$ and $z$ directions as well as in time after the statistically stationary state is reached. However, simulation of $b_{7}=0.9$ collapsed due to the numerical instability for which the reasons have been explained before; therefore, no statistical results of this case are presented.

The mean velocity profiles in inner and outer scaling are shown in Figure 9, in comparison with incompressible DNS of Moser et al. [43] and experimental measurements of Eckelmann [46] and Niederschulte et al. [47]. The van Driest transformed velocity $u_{V D}$ is used for the effective comparison between the compressible and incompressible results, according to the formula below,

$$
u_{V D}=\int_{0}^{\langle u\rangle} \bar{\rho} / \bar{\rho}_{W} d\langle u\rangle
$$

It can be seen from Figure 9 that the difference between all the profiles is small, which means the insensitive of the mean velocity prediction to the numerical dissipations tested within the MP7-LD scheme. In fact, all the schemes studied here are high-order and low-dissipation types; therefore, the correct mean flow predictions are always expected for these kinds of schemes. From detailed comparison of velocity profiles, we 
can observe a slight elevation of the velocity in the logarithmic region from the present simulations. Among the three tested cases, the results of the MP7-LD with $b_{7}=0.7$ and the COMP6 scheme are very close to each other, which indicates their similar performances in simulating wall turbulence.

(a)

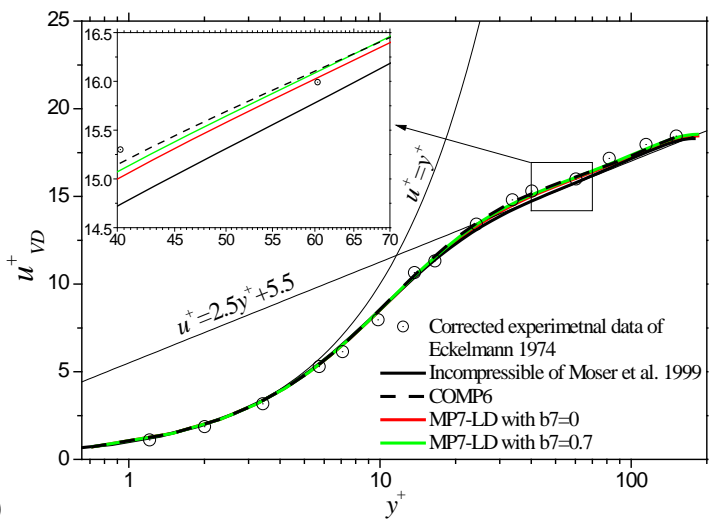

(b)

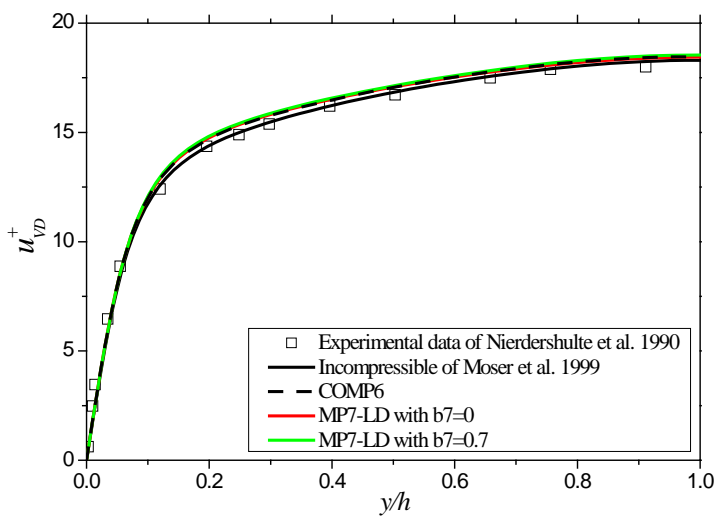

Figure 9. Mean velocity profiles in: (a) inner scaling and (b) outer scaling.

The RMS velocity fluctuations and the Reynolds shear stress in wall units are plotted in Figure 10(a).

Again, the difference between the test schemes and reference data is inconspicuous. For high-order statistics such as the RMS vorticity fluctuations shown in Figure 10(b), the improvement of using $b_{7}=0.7$ against $b_{7}=0$ is clearly visible, especially for the wall-normal component of the vorticity fluctuations $\omega_{y, \text { RMS }}$.

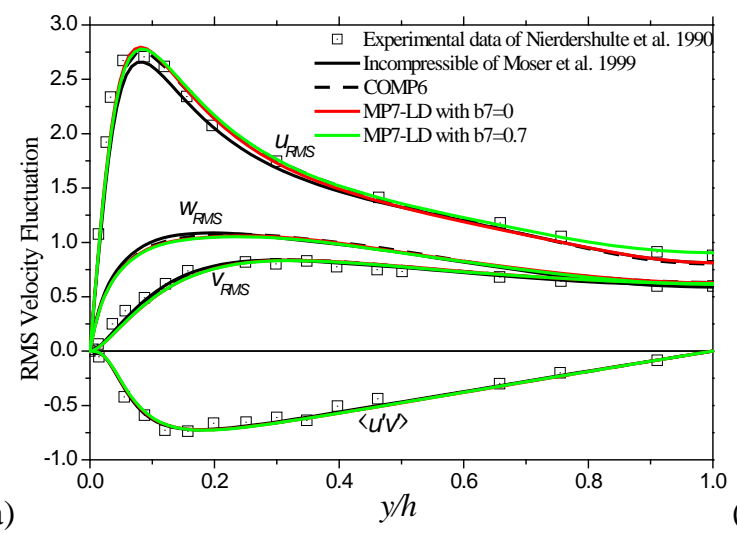

(a)

Figure 10. Comparison of (a) RMS velocity fluctuation and Reynolds shear stress and (b) RMS vorticity fluctuation. 
The effect of numerical dissipation is more salient on the prediction of turbulent velocity spectra as shown in Figure 11. In consistent with DNS of isotropic turbulence described before, the spectra of different schemes agree well with each other at the low wavenumber range. However, differences do exist at high wavenumbers. It can be seen that, the MP7-LD scheme with $b_{7}=0.7$ produces larger values of spectrum for all three velocity components than that of $b_{7}=0$, which means the better preservation of small-scale turbulence energy. The unphysical numerical oscillation has also been effectively suppressed, as there is no sign of tail-up of spectra curve at the end of the bandwidth for simulation using $b_{7}=0.7$.

(a)

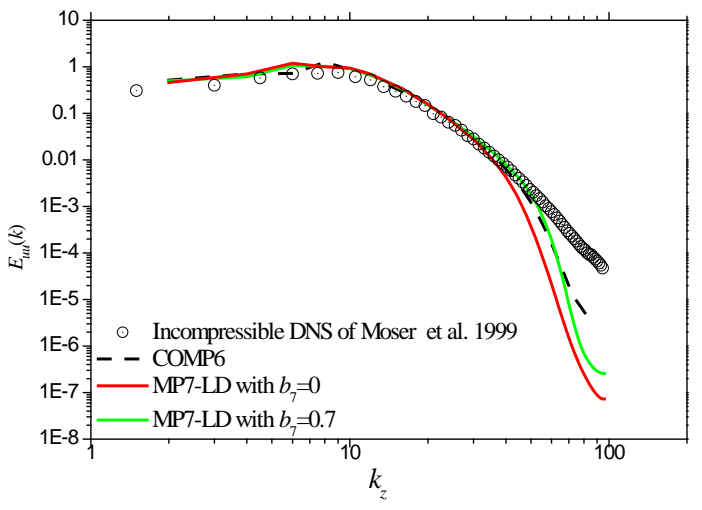

(b)

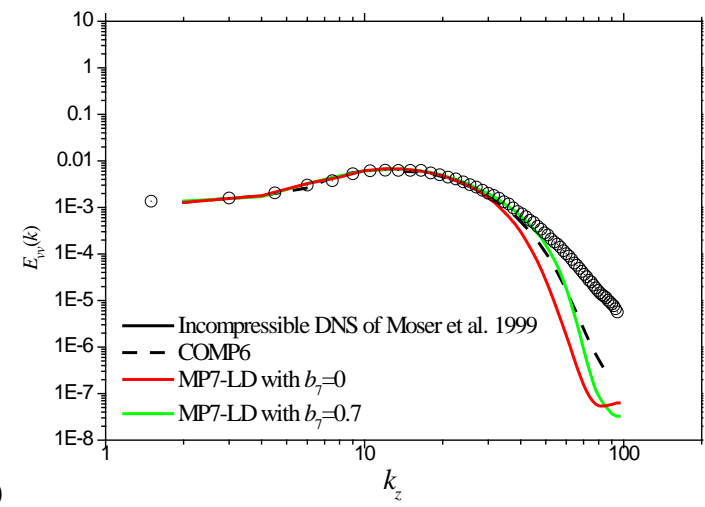

(c)

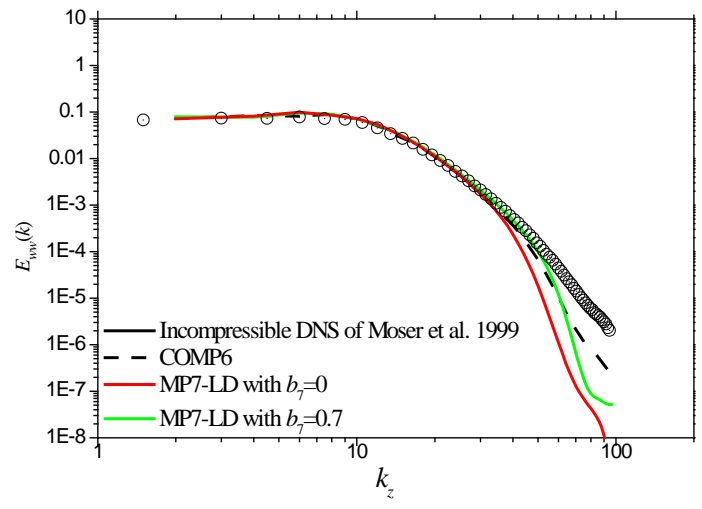

Figure 11. Spanwise velocity fluctuation spectra of (a) $u^{\prime \prime}$, (b) $v^{\prime \prime}$, (c) $w^{\prime \prime}$ at $y^{+}=10$. 
The small-scale turbulence coherent structures visualized by the iso-surface of $Q=\left(u_{0} / h\right)^{2}$ from four different schemes are shown in Figure 12. For the MP7-LD scheme with $b_{7}=0.9$, the flow field shown is just before the computation breakdown, and for other three schemes, the coherent structures are shown at the same time unit of $t=200$. Comparing Figures 12(a) and 12(b), the coherent structures obtained from the lower numerical dissipation $b_{7}=0.7$ case are more prosperous, which indicates the predicted turbulence is more energetic. The turbulence simulated by the COMP6 scheme (see Figure 12(d)) is the most energetic, since it has the lowest dissipation among all the assessed schemes. In Figure 12(c), there are large numbers of smallscale structures clustered, which is obvious unphysical and will cause simulation crash. It is believe that these small-scale structures are the results of the accumulation of unphysical errors that cannot be 'washed away' due to such a low level of numerical dissipation at $b_{7}=0.9$. Therefore, it is concluded that, a certain level of numerical dissipation must be kept for DNS of wall-bounded turbulence, and based on numerical studied so far, parameter $b_{7}=0.7$ is highly recommended as the optimized value for the MP7-LD scheme. However, the robustness of the MP-LD scheme is related to the value of the $b_{7}$. Smaller values of $b_{7}$ should be used for flows with complex geometry and grid, in which stronger numerical errors could be induced. We've tried $b_{7}=0.7$ in a series of SWTBLI, including impinging shock-wave/flat plate boundary layer interaction (the results will be presented in the next section), supersonic compression corner, expansion/compression corner and transonic airfoil, and MP-7LD scheme with $b_{7}=0.7$ worked well in these cases. For more complex flows, the value of $b_{7}$ could be case depended. 
(a)

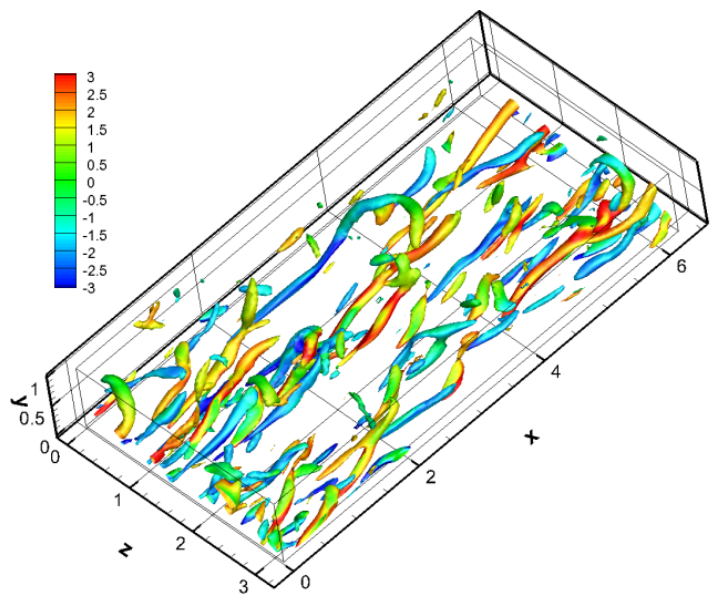

(c)

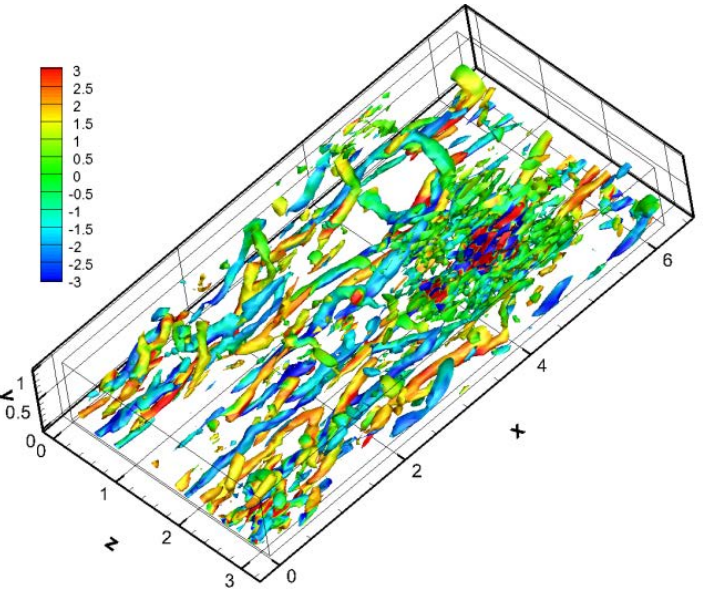

(b)

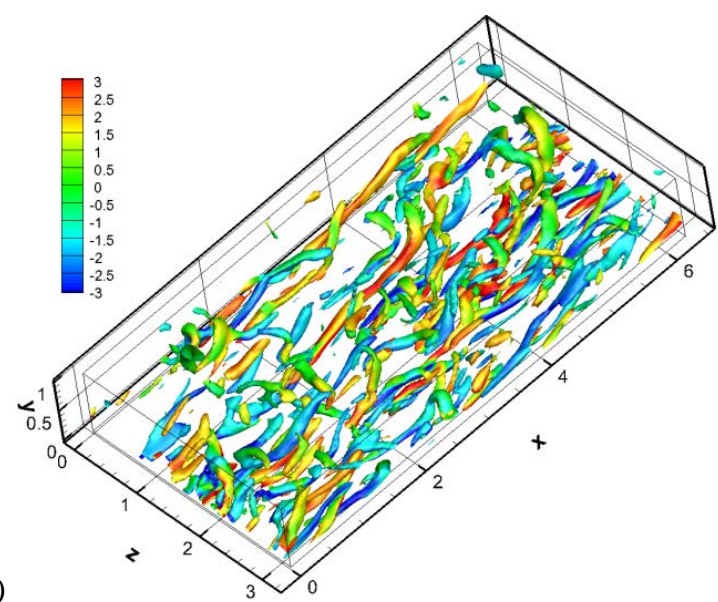

(d)

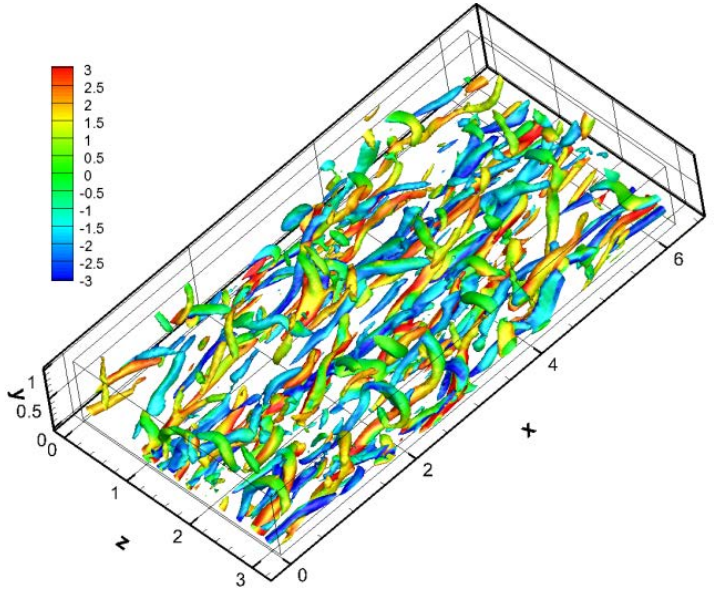

Figure 12. Turbulent coherent structures identified by $Q$ criterion and colored by streamwise vorticity. (a) MP7-LD with $b_{7}=0$; (b) MP7-LD with $b_{7}=0.7$; (c) MP7-LD with $b_{7}=0.9$; (d) COMP6.

\section{DNS of Shock-wave/Turbulent-Boundary-Layer Interaction}

By applying the optimized MP7-LD scheme with $b_{7}=0.7$, further study is conducted for DNS of SWTBLI to evaluate the performance of the MP7-LD scheme in simulating complex turbulent flows with shock-waves and separations.

\subsection{Computation Setup}

The flow configuration is sketched in Figure 13, in which the inlet plane is 4 inches from the leading-edge of the plate. The outlet plane is about 11 inches from the leading-edge and the inviscid shock impinging point 
at the wall is $x_{I}=10$ inches from the leading-edge. The Mach number of the incoming flow is $M=2.25$ and an oblique shock-wave at an angle of $33.2^{\circ}$, generated by a wedge, is introduced into the computational domain. The reference Reynolds number based on the reference length (1 inch) and velocity, density and viscosity of the incoming free-stream flow is $\mathrm{Re}=635000$, which corresponds to the zero-pressure-gradient flat plate boundary layer experimental test of Shutts et al. [48] (case 55010501). A laminar boundary layer profile based on compressible laminar boundary layer analysis is super-imposed at the inlet with the supersonic inflow boundary condition in the outer region and extrapolation of pressure in the subsonic portion of the boundary layer [49]. To trigger the boundary layer transition, the periodically wall blowing and suction boundary condition in the region of $4.5<x<5$ is used, which is the same as that of previous DNS of Rai et al. [50], Gao et al. [51], Pirozzoli and Grasso [52]. An adiabatic nonslip condition is used for the remaining wall surface. Near the outlet plane, an additional sponge zone with stretched grids and the second-order filter [53] is incorporated to drive the flow to a uniform state, and the supersonic outflow condition is used at the outlet plane. At the far field boundary of the upper surface of the computational domain, the single-point Rankine-Hugoniot relations are used to specify the free-stream values before and after the shock-wave.

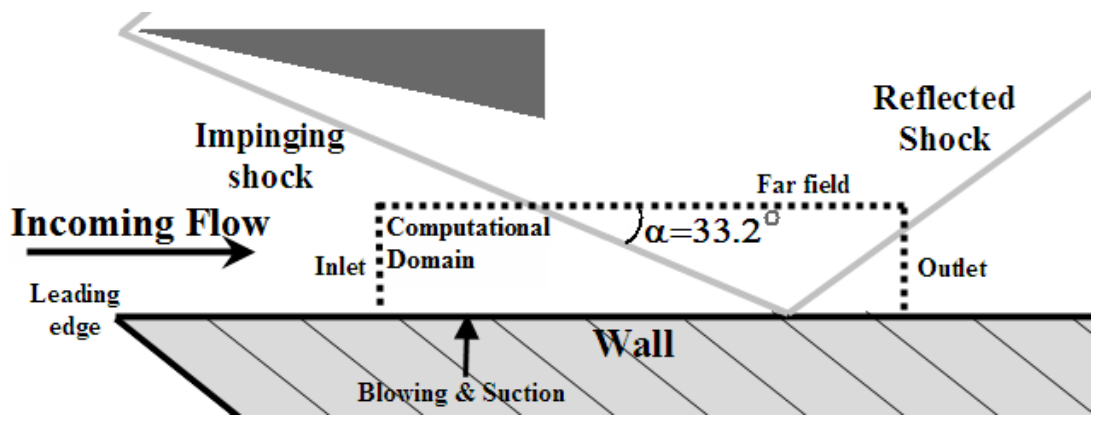

Figure 13 The sketch of computational domain of SWTBLI 
The domain size is $7.6 \times 1.2 \times 0.2$ and the orthogonal grid concentrating towards the wall and the interaction position is established. The grid has 2800, 150 and 256 points in streamwise, wall normal and spanwise directions, respectively. At the position immediately upstream the interaction zone ( $x=9.8)$, where the flow is fully developed with an equilibrium turbulent boundary layer status, the grid spacing are $\Delta x^{+}=3.9, \Delta y_{1}^{+} \approx$ $0.9, \Delta z^{+}=7.6$. The ratio of the effective grid spacing $\Delta=\sqrt[3]{\Delta x \Delta y \Delta z}$ to the local Kolmogorov length scale $\eta$ at $x=9.8$ and inviscid interaction position $x=10.0$ has been evaluated a posteriori (see Figure 14). It is evident that the effective grid spacing at these two key locations reaches the smallest turbulence length scale, which satisfies the resolution requirement for DNS.

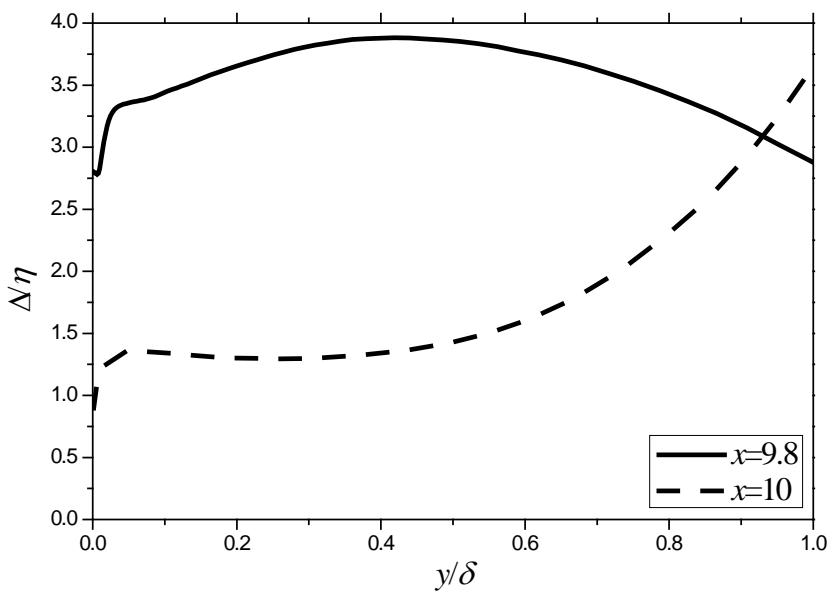

Figure 14. Profiles of the ratio of effective grid spacing to the local Kolmogorov length scale.

\subsection{Shock sensor}

The instantaneous distribution of shock sensor Eq. (19) at an $x-y$ plane is depicted in Figure 15, in which the oblique shock-wave impingement and reflection can be seen by the shock sensor function above the threshold of $\sigma=0.01$. Inside the wall boundary layer, where the turbulence is energetic, the MP limiter is completely deactivated, which ensures the turbulence being properly resolved at higher accuracy. 


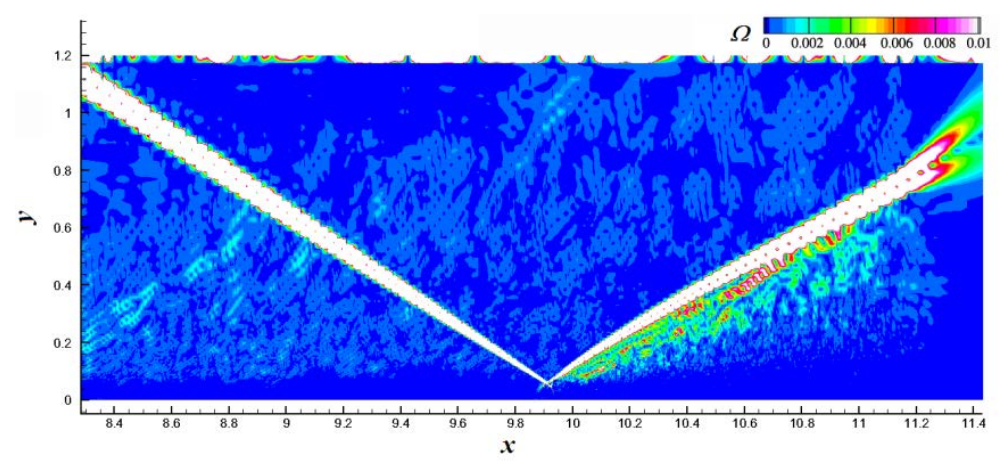

Figure 15. Distribution of shock sensor function.

\subsection{Skin Friction}

The mean wall skin friction $C f=\left.2 \operatorname{Re}\left\langle\mu_{W}\right\rangle \frac{\partial\langle u\rangle}{\partial y}\right|_{W}$ is plotted in Figure 16, in which the van Driest II transformation is used to extend the incompressible skin friction correlations to compressible flow conditions.

The expressions of the skin friction correlations of the incompressible laminar Blasius, turbulent Blasius and

Kármán-Schoenherr are respectively given as,

$$
\begin{gathered}
C f=0.6641 / \sqrt{\operatorname{Re}_{x}} \\
C f=0.026 / \sqrt{\operatorname{Re}_{\theta I}^{0.25}} \\
C f=1 /\left[17.08\left(\log \operatorname{Re}_{\theta I}\right)^{2}+25.11 \log \operatorname{Re}_{\theta I}+6.012\right]
\end{gathered}
$$

where $\operatorname{Re}_{\theta I}=\operatorname{Re}_{\theta} / \mu_{W}$, and $\theta$ is the momentum thickness of the boundary layer. 
(a)

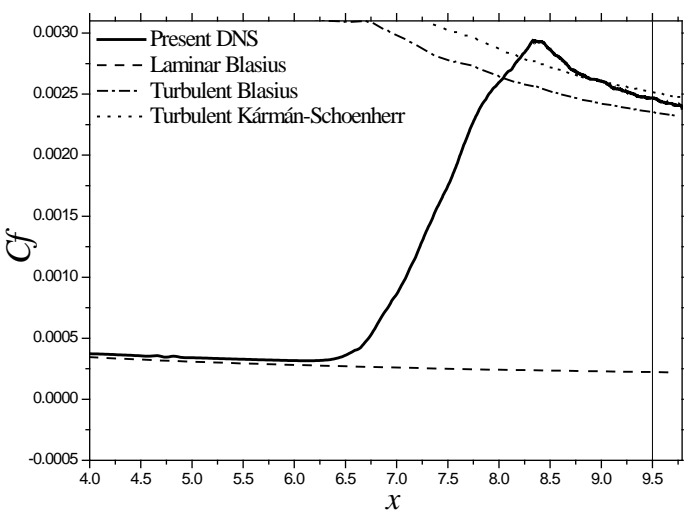

(b)

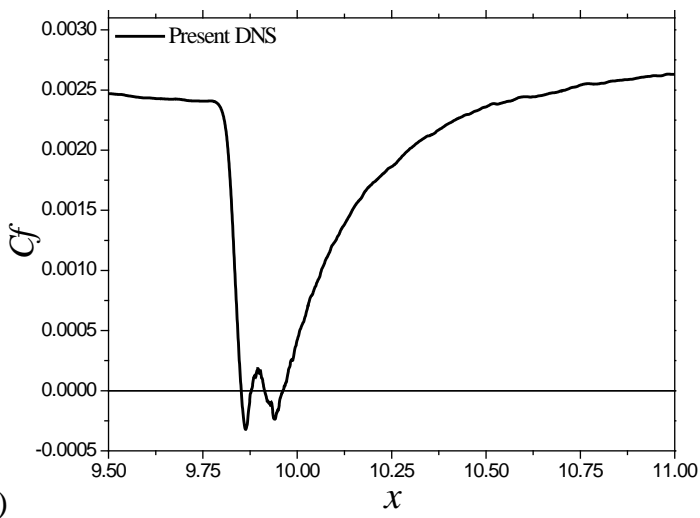

Figure 16. Wall skin friction coefficient distributions. (a) upstream the interaction region, and (b) in the interaction region.

According to Figure 16(a), a good agreement between the predicted skin friction coefficient and the laminar Blasius solution in the laminar boundary layer region can be seen. The increase of $C f$ after $x=6.25$ indicates the boundary layer transition occurred, and after reaching the maximum value near $x=8.25, C f$ falls back to a level between the turbulent Blasius and Kármán-Schoenherr correlations, which means the turbulent boundary layer is fully developed to an equilibrium status. After interacting with the shock-waves, $C f$ decreases steeply with negative values at $x=9.85$, which indicates the appearance of the reversed flow.

Downstream flow reattachment, the skin friction increases monotonously to a level even larger than that in the upstream location, which indicates the boundary layer is 'back' to turbulent boundary layer, but is still not yet fully recovered to an equilibrium status.

\subsection{Mean Flow}

The mean profile of fully developed equilibrium turbulent boundary layer at $x=9.5$ is analyzed. The boundary layer parameters are listed in Table 2, in which $\delta$ and $\delta^{*}$ are the $99 \%$ nominal boundary layer thickness and displacement thickness, and $\operatorname{Re}_{\tau}=\operatorname{Re} \rho_{W} u_{\tau} \delta / \mu_{W}$ is the friction Reynolds number. 
Table 2 Boundary layer parameters at $x=9.5$

\begin{tabular}{ccccc}
\hline \hline$C f$ & $\operatorname{Re}_{\delta}$ & $\operatorname{Re}_{\delta *}$ & $\operatorname{Re}_{\theta}$ & $\operatorname{Re}_{\tau}$ \\
\hline $2.47 \times 10^{-3}$ & 41167 & 11515 & 3148 & 685 \\
\hline \hline
\end{tabular}

The mean velocity profiles are plotted in outer and inner scalings in Figure 17(a) and 17(b), respectively.

The experimental measurements of Shutts et al. [48] and Bookey et al. [54] are also added in Figure 17(a) for comparison. The good agreements between the present DNS and experiments can be seen in the outer region of the boundary layer. In inner scaling, $u_{V D}^{+}$is highly coincident with the classic law of wall in both the linear sub-layer and log-layer. A good agreement between the present DNS and incompressible DNS of flat-plate turbulent boundary layer of Wu and Moin [55] at $\operatorname{Re}_{\theta}=900$ can also be found in Figure 17(b), except for the wake layer, where the present DNS shows stronger strength of the wake component due to the higher Reynolds number of the present case.
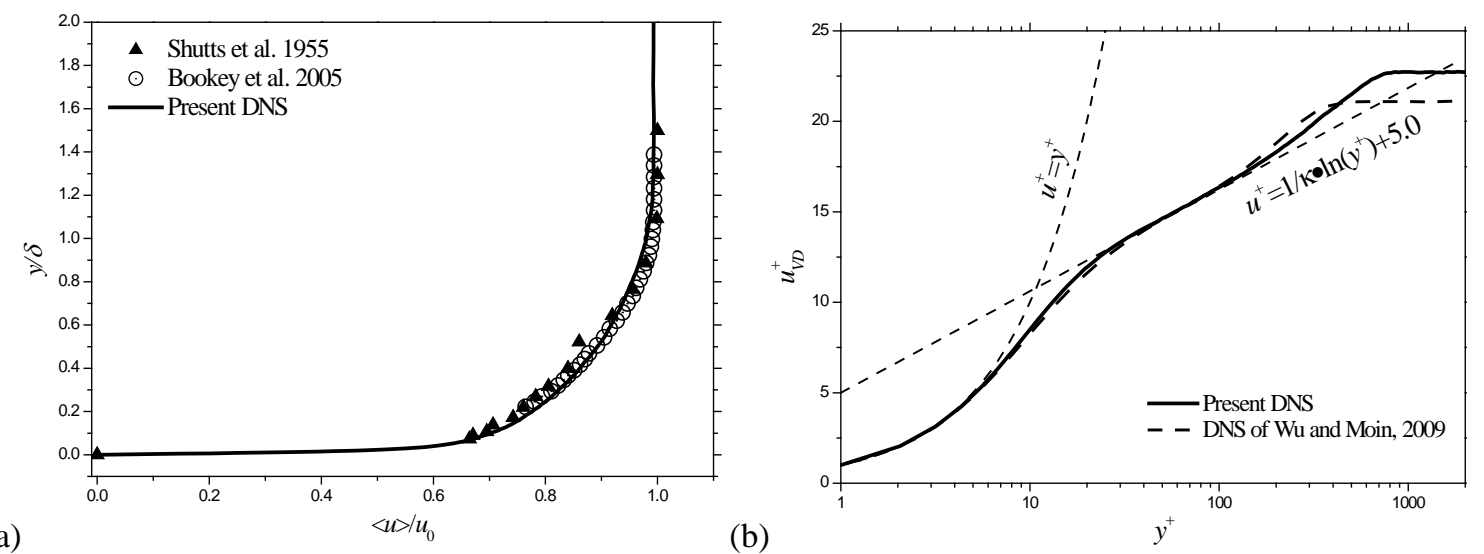

Figure 17. Mean velocity profile in (a) outer scaling and (b) inner scaling at $x=9.5 . \kappa=0.41$ is the von Kármán constant.

The mean temperature and mass flux profiles at $x=9.5$ are compared with the experimental data of Shutts et al. [48] and DNS result of Pirozzoli et al. [52] in Figure 18. Again, the present DNS results are in good agreement with the experimental data as well as the previous DNS at similar flow conditions. 


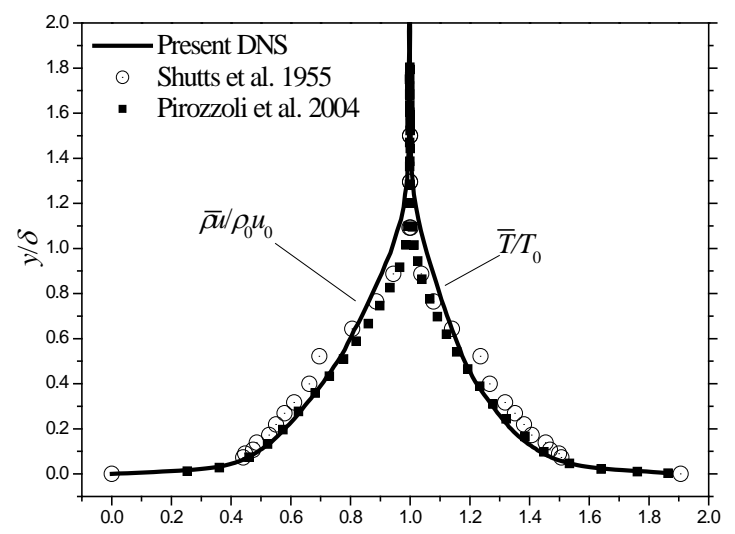

Figure 18. Profiles of mean temperature and mass flux at $x=9.5$.

The mean wall pressure in the interaction region is plotted in Figure 19, together with the experimental data of Dupont et al. [56] at two closest flow conditions ( $\mathrm{M}=2.3, \alpha=32.4$ and $\alpha=33.2)$, where $X^{*}$ and $P^{*}$ are defined in the same way as Dupont et al. [56], i.e. $X^{*}=\left(x-X_{0}\right) / L_{S}, P^{*}=\left(\bar{p}-p_{1}\right) /\left(p_{2}-p_{1}\right)$, where $X_{0}$ is the mean position of the reflected shock foot, $L_{S}$ is the length of the interaction zone, $p_{1}$ and $p_{2}$ are the pressure upstream and downstream of the impinging shock deduced from the inviscid theory.

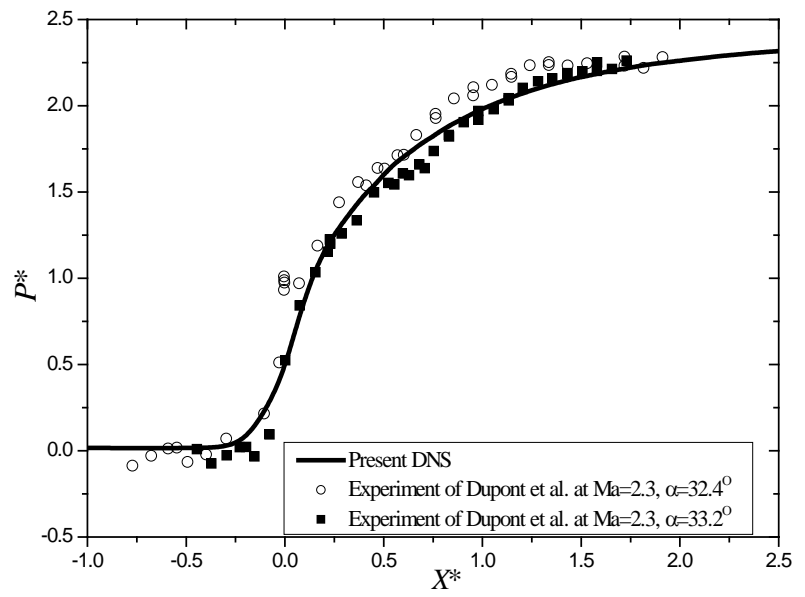

Figure 19. Mean wall pressure distributions in comparison with experiments.

It can be seen that the mean wall pressure of the present DNS matches well with the measurement data.

The predicted wall pressure is increased smoothly, because the compression in the near-wall region is carried out by a series of compression waves due to strong inviscid-viscous interactions. 
Figure 20 compares the mean and instantaneous flow field with a recent PIV experiment of Humble et al.

$[57,58]$ at $\mathrm{M}=2.07, \operatorname{Re}_{\theta}=4.92 \times 10^{4}$ and $\alpha=35.4^{\circ}$. The validation is merely qualitatively due to the

difference in flow conditions. To be consistent with the experiment, the coordinates in Figure 20 are

normalized as $x^{*}=\left(x-x_{I}\right) / \delta_{\text {ref }}$ and $y^{*}=y / \delta_{\text {ref }}$, in which $\delta_{\text {ref }}$ is the nominal boundary layer thickness

at $x^{*}=-3$.

(a)

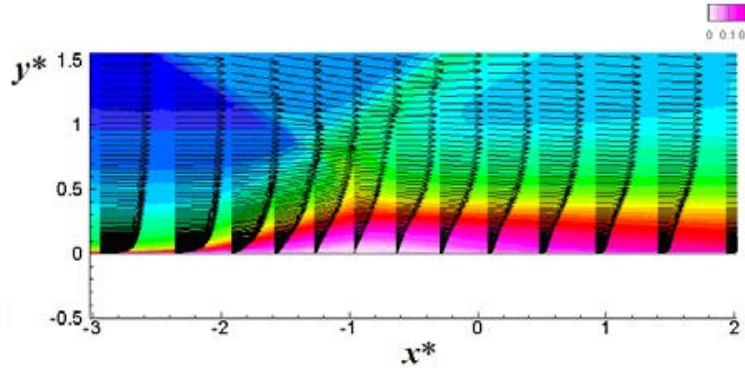

(b)

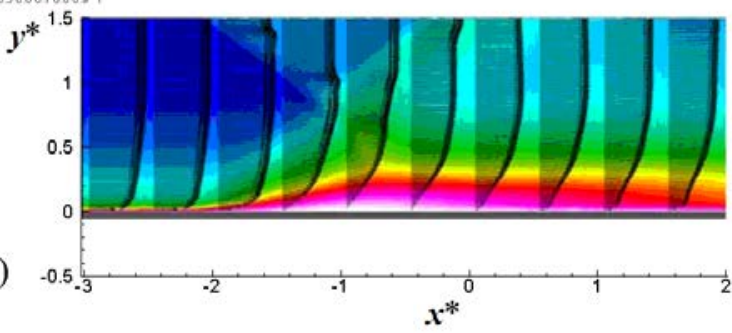

(c)

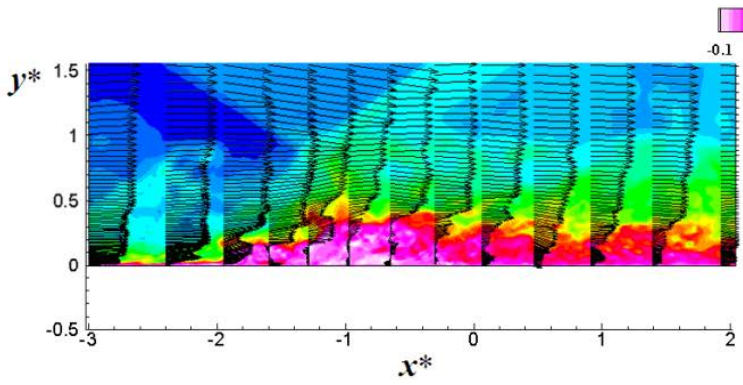

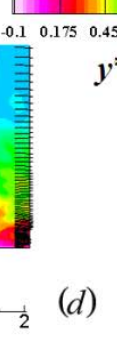

(d)

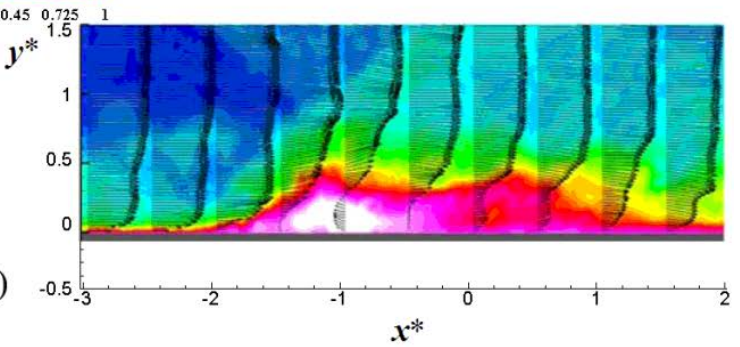

Figure 20. Mean (a, b) and instantaneous (c, d) streamwise velocity and velocity vector s of present DNS (a, c) and PIV measurement of Humble et al. [57] (b, d).

It can be seen from Figure 20 that DNS results and PIV measurements are very similar in terms of mean

and instantaneous flow patterns, including the thickening of the boundary layer after interacting with the

shock-wave, the formation of the mixing layer during the interaction as well as the complex instantaneous

reverse flow in the interaction region. Both flows show small separation; however, from the Figure 20 (c, d),

we can see the instantaneous flow separation happens in much larger region than that of the mean flow, which 
indicates the strong unsteadiness of the flow separation. Comparing with the PIV measurement, the DNS captures more flow details in the near-wall and the interaction regions due to its higher spatial resolution.

\subsection{Turbulence Statistics}

According to the Morkovin's hypothesis, we compared the density scaled RMS velocity fluctuations $u_{i, D R M S}^{\prime \prime}=\sqrt{\frac{\bar{\rho}}{\overline{\rho_{W}}}\left\langle u_{i}^{\prime \prime} u_{i}^{\prime \prime}\right\rangle}$ at location of $x=9.5$ before turbulence/shock interaction with low Reynolds number boundary layer experimental data of Purtell at al. [59] and Erm and Joubert [60], incompressible DNS of Spalart [61] and Wu and Moin [55], as well as a recent compressible DNS of Pirozzoli et al. [62] in Figure 21, in which $u_{i, D R M S}^{\prime \prime}$ is normalized with the wall friction velocity.
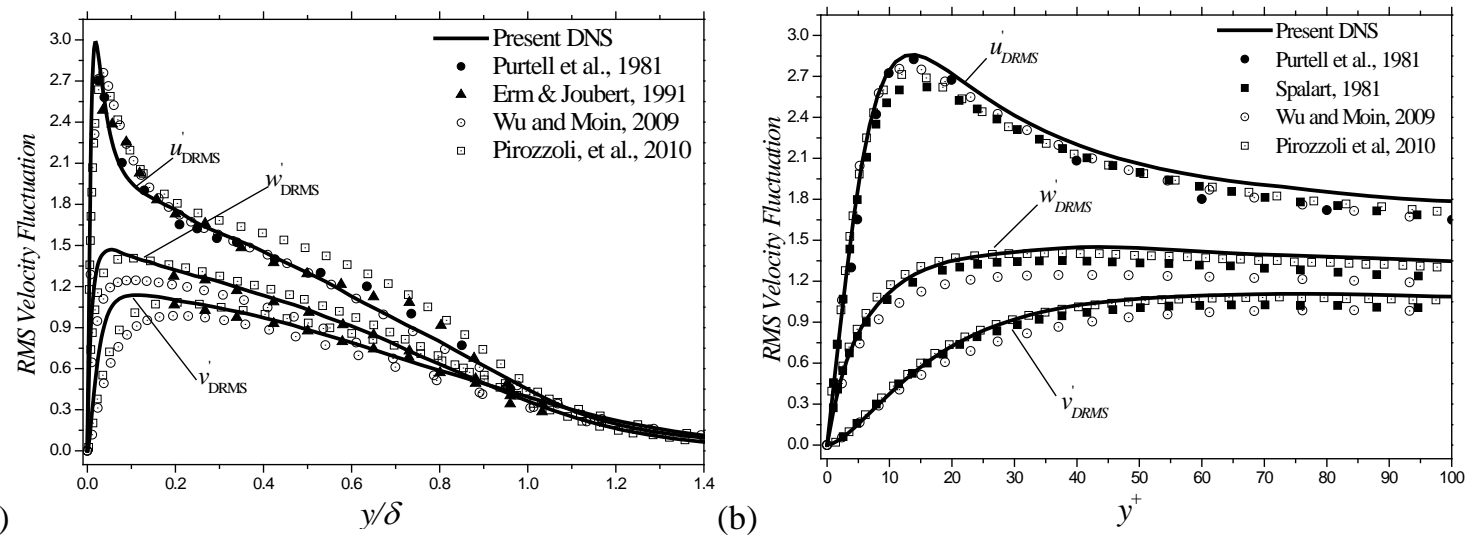

Figure 21. Density scaled RMS velocity fluctuation in outer scaling (a) and inner scaling (b) at $x=9.5$.

In the outer region of the turbulent boundary layer, good agreements have been achieved for all the RMS

velocity fluctuation components compared with the experimental data and other DNS results. In the inner (near-wall) region, better agreements among all these data are obtained, due to the smaller influence of the flow Reynolds number on turbulence statistics in the near-wall region when the inner scaling is adopted. The 
peak value of streamwise velocity fluctuation attains 2.86 at $y^{+}=14$, in good agreement with experimental data of Purtell [59].

The comparison of the streamwise and the wall-normal velocity fluctuation intensities: $\sqrt{\left\langle u^{\prime \prime} u^{\prime \prime}\right\rangle}$ and $\sqrt{\left\langle v^{\prime \prime} v^{\prime \prime}\right\rangle}$ in the interaction region between the present DNS and PIV of Humble et al. [57] is given in Figure 22, and qualitative agreements can be found, including the amplification of the velocity intensities and the change of their distribution patterns during the interaction. According to Figure 22, in both DNS and experiment, the turbulence begins to be amplified at about $2 \delta$ upstream the inviscid interaction point $x_{I}$. In the equilibrium region, the turbulence energy is more concentrated in the near-wall region, which can also be confirmed in Figure 21. The interaction with the shock-wave greatly enhances the fluctuation intensities, drafts their peaks away from the wall and changes their distribution patterns, which can be partly due to the formation of the mixing layer. [63, 64] Therefore, downstream the interaction region, there is a wide region with strong fluctuations and the peaks of both $\sqrt{\left\langle u^{\prime \prime} u^{\prime \prime}\right\rangle}$ and $\sqrt{\left\langle v^{\prime \prime} v^{\prime \prime}\right\rangle}$ move from the near-wall region to $y \approx 0.3 \delta_{\text {ref }}$, where a 'core' of the mixing layer locates. Further downstream, the velocity intensities in the outer region of boundary layer are gradually damped out due to the decay of the mixing layer and the damping rate in the experiment is somehow faster than that of the present DNS. In experiment, the near-wall peak of $\sqrt{\left\langle u^{\prime \prime} u^{\prime \prime}\right\rangle}$ begins to recover (see Figure 22 (b)), which indicates the regeneration of the wall turbulence, however, this phenomenon happens at a further downstream location in the present DNS, which could be attributed to the lower Reynolds number of the present study. 
(a)

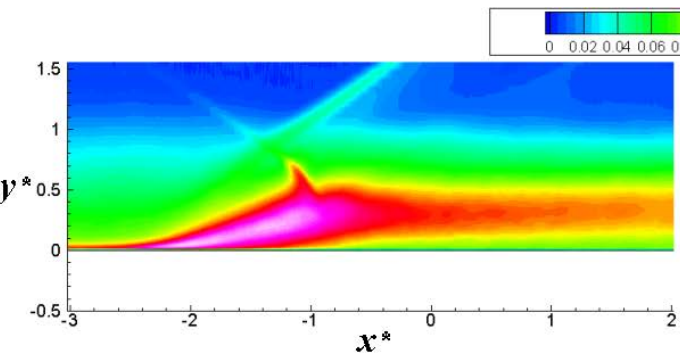

(b)

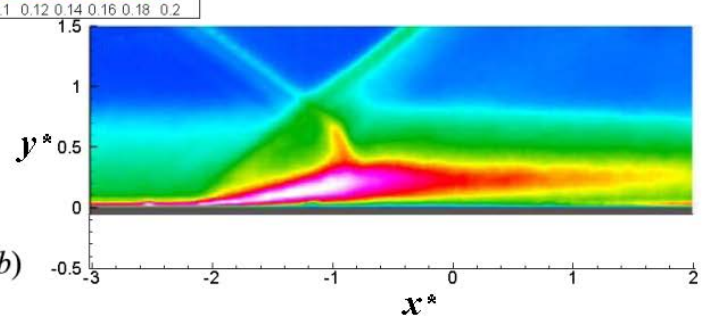

$(c)$
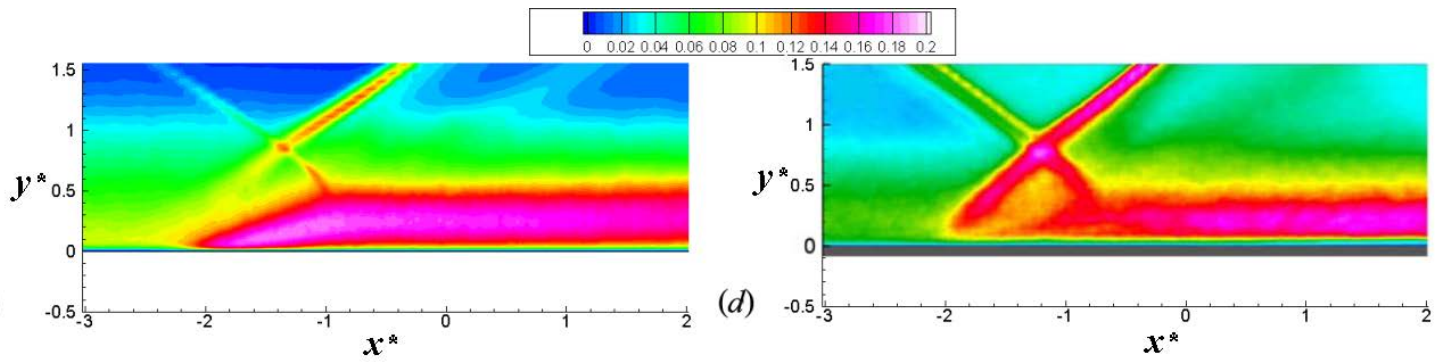

Figure 22. Velocity fluctuation intensities in the interaction region, $\sqrt{\left\langle u^{\prime \prime} u^{\prime \prime}\right\rangle}(a, b)$ and $3 \sqrt{\left\langle v^{\prime \prime} v^{\prime \prime}\right\rangle}$ (c, d) normalized with the velocity in the incoming flow of the present DNS (a, c) and PIV measurement of Humble et al. [57] (b, d).

The instantaneous turbulence coherent structures are visualized in Figure 23 by using the iso-surface of

the swirling strength $\lambda_{c i}$ [65] equaling to $0.5 \%$ of its global maximum, from which it can be seen the turbulence coherent structures are well resolved by using the optimized MP7-LD scheme and the shock-wave is also well captured. The dramatic change in turbulent structures while passing through the shock-wave can also be observed. In the undisturbed turbulent boundary layer, the streamwise elongated coherent structures in the near wall region, known as horseshoe vortex or hairpin vortex $[65,66]$ are predominant. After interacting with the shock-wave, the turbulent flow are detached from the wall, resulting in a thicker layer with much chaotic characteristics, which indicates the change of the turbulence production mechanism from a wallbounded turbulence to a free shear-layer turbulence. The large-scale deformation of the impinging shock- 
wave surface can also be seen (pointed with an black arrow), which is consistent with the observation by

Priebe et al. [64].

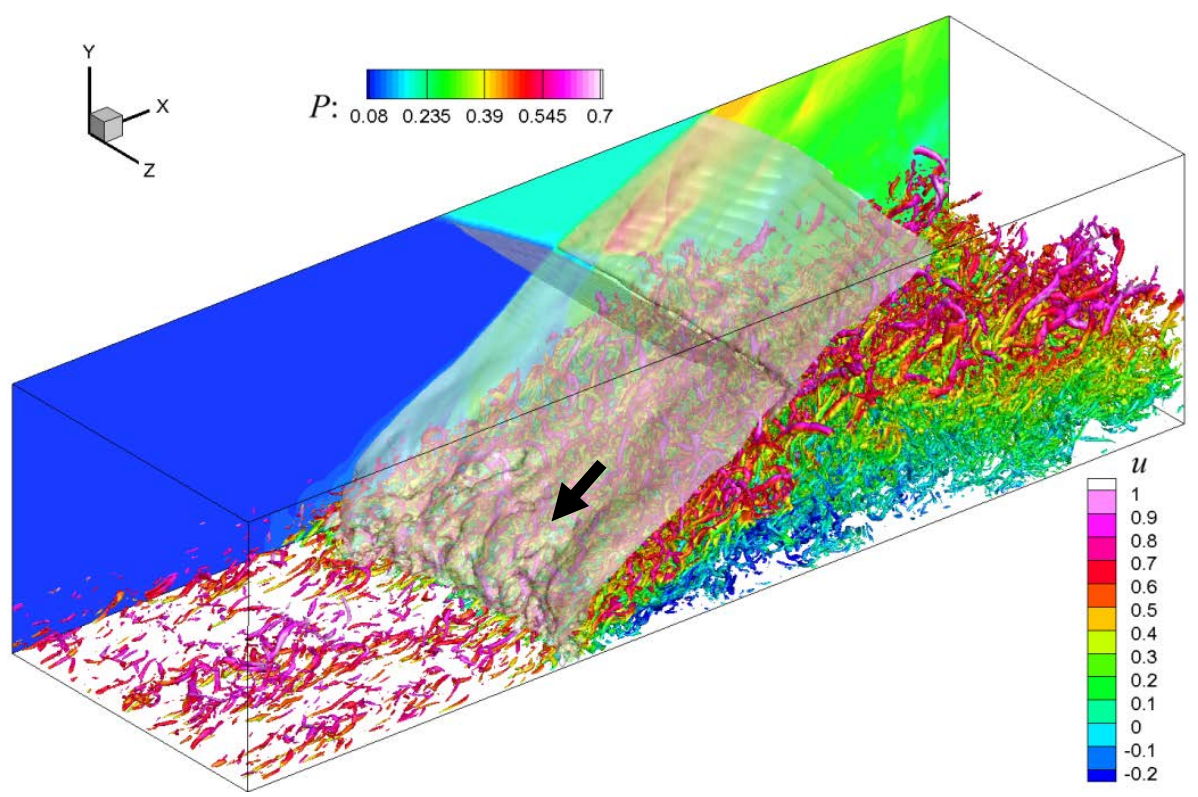

Figure 23. Turbulence coherent structures visualized using iso-surface of $\lambda_{c i}$, rendered with the instantaneous streamwise velocity. The shock surface is visualized by using the iso-surface of the pressure gradient and a slice of pressure field is also shown.

\subsection{Turbulent Kinetic Energy Budget}

The turbulent kinetic energy transport equation can provide critical information for investigations of

turbulence mechanisms and modelling and it can also be used to validate a DNS by checking the balance of

the TKE transport equation [67]. The TKE transport equation is expressed as [62],

$$
\frac{\partial 0.5 \bar{\rho}\left\langle u_{k}^{\prime \prime} u_{k}^{\prime \prime}\right\rangle}{\partial t}=C+T+P+V-\varepsilon+K
$$

where, $C=-\frac{\partial}{\partial x_{j}}\left[0.5 \bar{\rho}\left\langle u_{j}\right\rangle\left\langle u_{k}^{\prime \prime} u_{k}^{\prime \prime}\right\rangle\right]$ is the advection term, $T=-\frac{\partial}{\partial x_{j}}\left[0.5 \bar{\rho}\left\langle u_{i}^{\prime \prime} u_{i}^{\prime \prime} u_{j}^{\prime \prime}\right\rangle+\overline{p^{\prime} u_{\jmath}^{\prime \prime}}\right]$ is the turbulent transport term, $P=-\bar{\rho}\left\langle u_{i}^{\prime \prime} u_{j}^{\prime \prime}\right\rangle \frac{\partial\left\langle u_{i}\right\rangle}{\partial x_{j}}$ is the production term, $V=\frac{\partial}{\partial x_{j}}\left(\overline{\tau_{l \jmath}^{\prime} u_{\imath}^{\prime \prime}}\right)$ is the viscous diffusion term, 
$\varepsilon=\overline{\tau_{l_{\jmath}}^{\prime} \frac{\partial u_{l}^{\prime \prime}}{\partial x_{\jmath}}}$ is the dissipation term and $K=\overline{p^{\prime} \frac{\partial u_{l}^{\prime \prime}}{\partial x_{\jmath}}}+\overline{\overline{u_{l}^{\prime \prime}}}\left(\frac{\partial \overline{\bar{\tau}_{l}}}{\partial x_{j}}-\frac{\partial \bar{P}}{\partial x_{i}}\right)$ is the term accounting for the direct effect of compressibility through pressure-dilatation correlation and mass diffusion.

The distributions of all terms in the undisturbed boundary layer at $x^{*}=-3$ are shown in Figure 24, in which all terms are normalized with $\rho_{W}^{2} u_{\tau}^{4} / \mu_{W}$. The data from DNS of SWTBLI of Pirozzoli and Bernardini [68] at Mach=2.28 are given for comparison. In the most part of the undisturbed boundary layer $\left(y^{+}>30\right)$, the transport equation presents the balance between the production and dissipation. The viscous diffusion and turbulent transport terms become effective only in the near-wall region, which transport TKE from high production region towards the wall. The production term maximizes at about $y^{+}=12$, which is also the peak position of streamwise velocity fluctuation and indicates the existence of organized turbulent structures as presented in Figure 23. The dissipation terms increases when approaching the wall, and is balanced by the viscous diffusion term at the wall. The present DNS agrees well with the data of Pirozzoli and Bernardini [68] and the balance calculated as the sum of all terms in Eq. (35), is very close to zero, which means the DNS is well resolved in the undisturbed boundary layer.

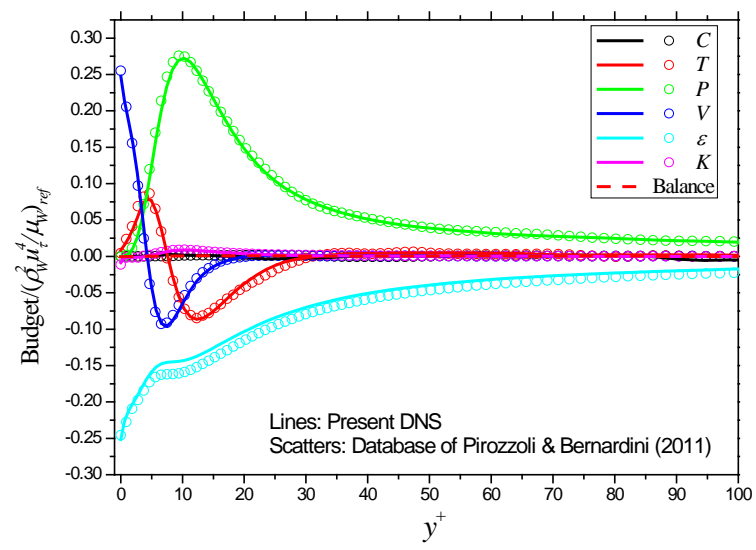

Figure 24. Turbulence kinetic energy balance at $x^{*}=-3$ 
The distributions of TKE budgets normalized with $\rho_{W}^{2} u_{\tau}^{4} / \mu_{W}$ at $x^{*}=-3$ in the interaction region are shown in Figure 25. It can be seen that, all terms are greatly changed in the interaction zone and their relation becomes complex. Therefore, the balance of all terms is a challenge to the numerical method of the DNS. The production and dissipation terms are greatly increased in the mixing layer generated in the interaction region, in which the turbulence is dominated by some large-scale turbulent coherent structures as shown in Figure 23. The strong TKE production in the mixing layer is balanced by the turbulent transport and dissipation terms.

The viscous diffusion term is restricted in the very near-wall region, even in the interaction region. The term

$K$ is effective in regions with shock-waves and compression waves, where compressibility is strong.

(a)

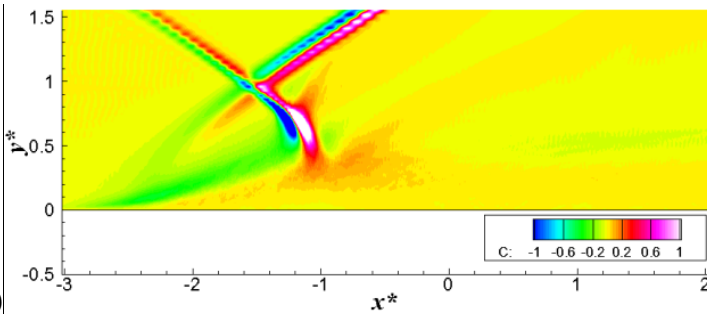

(c)

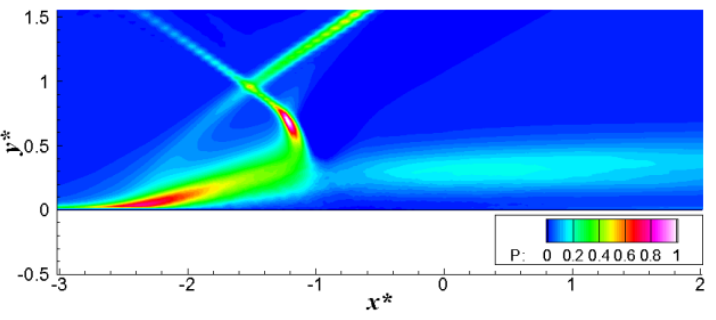

(e)

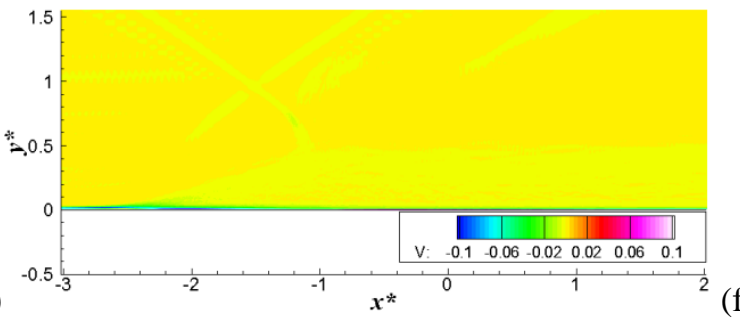

(b)

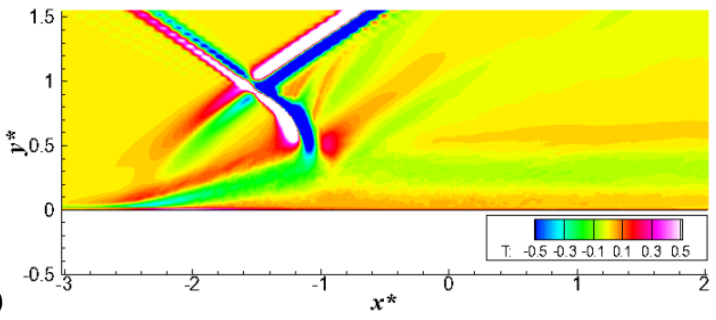

(d)

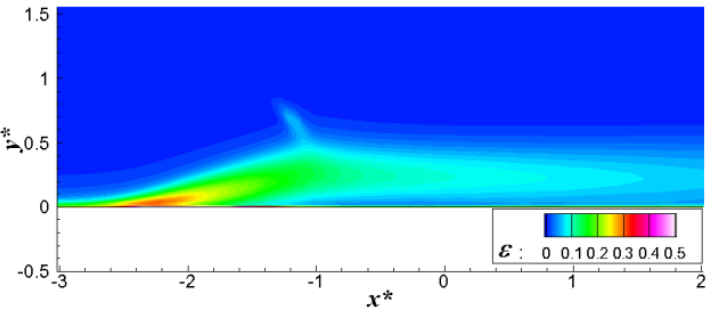

(f)

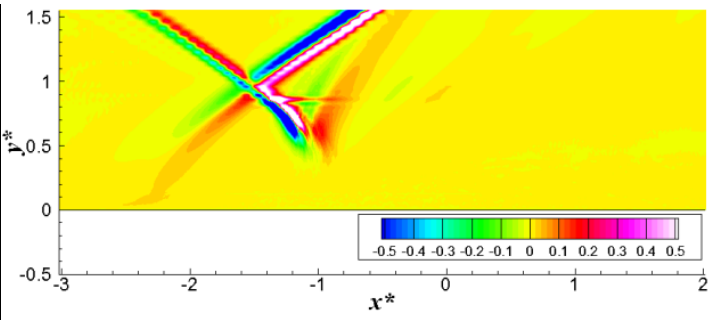

Figure 25. Turbulence kinetic energy budget terms. (a): $C$, (b): $T$, (c): $P,(d): \varepsilon,(e): V$, (f): $K$ 
The profiles of terms of the TKE transport equation in the interaction $\left(x^{*}=-0.21\right)$ and recovery regions $\left(x^{*}=9.0\right)$ are shown in Figure 26, in which $y^{+}$is calculated with the wall values at $x^{*}=-3$. The DNS data of Pirozzoli and Bernardini [68] at similar locations are also plotted. The quantitative comparison between the present DNS and the data of Pirozzoli and Bernardini [68] is poor, since the flow in the interaction and recovery region is in the state of non-equilibrium. However, we can still identify the consistent trend of all terms between the two DNS. In the interaction region of $x^{*}=-0.21$, the strong production is in the mixing layer and is balanced by dissipation and turbulent transport. TKE is transported towards the wall by the turbulent transport term and the near-wall TKE transport is still accomplished by the viscous diffusion term, which are balanced by the dissipation term. In the recovery region, the production term decreases in outer part of the boundary layer and increases in the near-wall region, which indicates the decay of the mixing layer and regeneration of wall turbulence. All other terms become similar with those in the undisturbed boundary layer, which indicates the recovery of the boundary layer towards the state of equilibrium. Although all terms change greatly in the interaction region, the balance of the TKE transport equation is well preserved, which means the good performance of the present MP-LD scheme in the DNS.
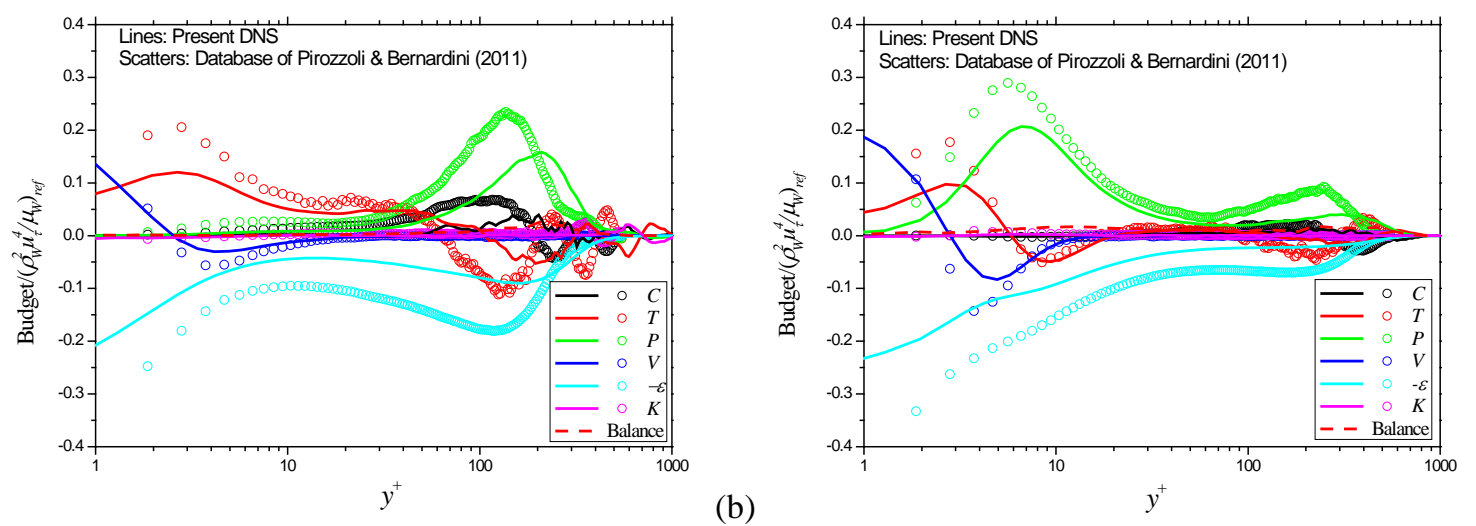

Figure 26. Turbulence kinetic energy balance at $x^{*}=-0.21$ (a) and $x^{*}=9$ 


\section{Concluding Remarks}

This paper investigated the influences of numerical dissipation of the MP7-LD scheme on DNS of both isotropic turbulence and wall-bounded turbulence and an optimized value of the parameter $b_{7}=0.7$ has been derived and confirmed during benchmark tests. The DNS of the impinging shock-wave/turbulent-boundarylayer interaction is then carried out by using the optimized MP7-LD scheme and the results are well validated against available experimental data and other DNS results, which prove the applicability of the MP7-LD scheme for DNS of complex turbulent flows interacting with shock-waves. Some conclusions can be drawn as follows,

(1) The numerical dissipation influences turbulence simulation mainly on two aspects: first, the excessive numerical dissipation will suppress small-scale turbulent fluctuations and over-dissipate turbulent energies, therefore, reduces the accuracy of DNS/LES; second, if the numerical dissipation is too low, the unphysical numerical error in terms of small-scale oscillations could develop and grow, which will contaminant the simulation results, and even destroy the computations.

(2) An optimized level of numerical dissipation should be kept for stable DNS computation. For the MP7LD scheme, the parameter $b_{7}=0.7$, which reduces $70 \%$ of the bandwidth dissipation of the conventional seventh-order upwind scheme, is recommended as the optimized value for DNS of both isotropic turbulence and wall-bounded turbulence, according to case studies in the present research. It is also found that, for highorder DNS of wall-bounded turbulence with a sufficient finer grid resolution, the influence of bandwidth dissipation is mainly presented in the spectra at the high wavenumber range. It has little influence on the prime statistics, such as the mean flow field and the Reynolds stresses profiles. 
(3) The optimized MP7-LD scheme can be efficiently applied in DNS of complex turbulent flows with shock interactions and shock-induced flow separations. Both the detailed turbulent structures and accurate flow statistics can be acquired. The turbulence is amplified with a dramatic change of the coherent structures during the interaction region, which indicates the change of turbulence production mechanism from fully attached wall turbulence to detached mixing layer. The analysis of the TKE transport equation further confirms the high TKE production in the mixing layer. The terms of the TKE transport equation are greatly changed and the relation among all terms becomes complex in interaction region. The balance of the equation is still well preserved in all the regions, which proves the good resolution of the present DNS.

\section{Acknowledgments}

This work is supported by the National Natural Science Foundation of China (11302012, 51136003, 50976010, 51006006), China Postdoctoral Science Foundation, the National Basic Research Program of China (2012CB720205), National Magnetic Confinement Fusion Research Program of China (2012GB102006), the Aeronautical Science Foundation of China (2010ZB51025), the 111 Project (B08009), and the Astronautical Technology Innovation Foundation of China. Part of the computer time for the present study was provided via the UK Turbulence Consortium (EPSRC grant EP/G069581/1).

\section{References}

[1] Pirozzoli S. Numerical Methods for High-Speed Flows. Annual Review of Fluid Mechanics. 2011;43:16394.

[2] Sandham ND, Li Q, Yee HC. Entropy Splitting for High-Order Numercial Simulation of Compressible Turbulence. Journal of Computational Physics. 2002;178:307-22.

[3] Kennedy CA, Gruber A. Reduced aliasing formulations of the convective terms within the Navier Stokes equations for a compressible fluid. Journal of Computational Physics. 2008;227:1676-700. 
[4] Touber E, Sandham ND. Large-eddy simulation of low-frequency unsteadiness in a turbulent shockinduced separation bubble. Theoretical and Computational Fluid Dynamics. 2009;23:79-107.

[5] Larsson J, Lele SK. Direct numerical simulation of canonical shock/turbulence interaction. Physics of Fluids. 2009;21:126101-12.

[6] Larsson J, Gustafsson B. Stability criteria for hybrid difference methods. Journal of Computational Physics. 2008;227:2886-98.

[7] Pirozzoli S. Conservative Hybrid Compact-WENO Schemes for Shock-Turbulence Interaction. Journal of Computational Physics. 2002;178:81-117.

[8] Shu CW, Osher S. Efficient Implementation of Essentially Non-oscillatory Shock-Capturing Schemes. Journal of Computational Physics. 1988;77:439-71.

[9] Shu CW, Osher S. Efficient Implementation of Essentially Non-oscillatory Shock-Capturing Schemes, II. Journal of Computational Physics. 1989;83:32-78.

[10] Jiang GS, Shu CW. Efficient Implementation of Weighted ENO Schemes. Journal of Computational Physics. 1996;126:202-28.

[11] Johnsen E, Larsson J, Bhagatwala AV, Cabot WH, Moin P, Olson BJ, et al. Assessment of highresolution methods for numerical simulations of compressible turbulence with shock waves. Journal of Computational Physics. 2010;229:1213-37.

[12] Garnier E, Mossi M, Sagaut P, Comte P, Devilley M. On the Use of Shock-Capturing Schemes for Large-Eddy Simulation. Journal of Computational Physics. 1999;153.

[13] Martin MP, Taylor EM, Wu M, Weirs VG. A bandwidth-optimized WENO scheme for the effective direct numerical simulation of compressible turbulence. Journal of Computational Physics. 2006;220:270-89.

[14] Borges R, Carmona M, Costa B, Don WS. An improved weighted essentially non-oscillatory scheme for hyperbolic conservation laws. Journal of Computational Physics. 2008;227:3191-211.

[15] Wang ZJ, Chen RF. Optimized Weighted Essentially Nonoscillatory Schemes for Linear Waves with Discontinuity. Journal of Computational Physics, Volume. 2001;174:381-404.

[16] Zahran YH, Babatin MM. Improved ninth order WENO scheme for hyperbolic conservation laws. Applied Mathematics and Computation. 2013;219:8198-212.

[17] He Z, Li X, Liang X. Nonlinear spectral-like schemes for hybrid schemes. Science China Physics, Mechanics and Astronomy. 2014;57:753-63.

[18] Tu GH, Yuan XJ. A characteristic-based shock-capturing scheme for hyperbolic problems. Journal of Computational Physics. 2007;225:2083-97.

[19] Tenaud VD, C. High order one-step monotonicity-preserving schemes for unsteady compressible flow calculations. Journal of Computational Physics. 2004;193:563-94.

[20] Rider WJ, Margolin LG. Simple Modifications of Monotonicity-Preserving Limiter. Journal of Computational Physics. 2001;174:473-88.

[21] Suresh A, Huynh HT. Accurate Monotonicity-Preserving Schemes with Runge Kutta Time Stepping. J Comput Phys. 1997;136:83-99.

[22] Christophe Bogey NdCC. A shock-capturing methodology based on adaptative spatial filtering for highorder non-linear computations. Journal of Computational Physics. 2009;228:1447-65. 
[23] Yee HC, Sandham ND, Djomehri MJ. Low-Dissipative High-Order Shock-Capturing Methods Using Characteristic-Based Filters. Journal of Computational Physics. 1999;150:199-238.

[24] Li Z, Jaberi FA. A high-order finite difference method for numerical simulations of supersonic turbulent flows. International Journal for Numerical Methods in Fluids. 2011;68:740-66

[25] Jammalamadaka A, Li Z, Jaberi FA. Subgrid-Scale Models for Large-Eddy Simulations of ShockBoundary-Layer Interactions. AIAA Journal. 2013;51:1174-88.

[26] Jammalamadaka A, Li Z, Jaberi FA. Large-Eddy Simulation of Turbulent Boundary Layer Interaction with an Oblique Shock Wave. 48th AIAA Aerospace Sciences Meeting: AIAA Paper 2010-110; 2010. [27] Li X-l, Leng Y, He Z-w. Optimized sixth-order monotonicity-preserving scheme by nonlinear spectral analysis. International Journal for Numerical Methods in Fluids. 2013;73:560-77.

[28] Fang J, Li Z, Lu L. An Optimized Low-Dissipation Monotonicity-Preserving Scheme for Numerical Simulations of High-Speed Turbulent Flows. Journal of Scientific Computing. 2013;56:67-95.

[29] Ekaterinaris JA. High-order accurate, low numerical diffusion methods for aerodynamics. Progress in Aerospace Sciences. 2005;41:192-300.

[30] Ducros F, Ferrand V, Nicoud F, Weber C, Darracq D, Gacherieu C, et al. Large-Eddy Simulation of the Shock/Turbulence Interaction. Journal of Computational Physics. 1999;152:517-49.

[31] Lo SC, Blaisdell GA, Lyrintzis AS. High-order shock capturing schemes for turbulence calculations. International Journal for Numerical Methods in Fluids. 2009;62:473-98.

[32] Visbal MR, Gaitonde DV. Shock Capturing Using Compact-Differencing-Based Methods. 43rd AIAA Aerospace Sciences Meeting and Exhibit. Reno. NV: AIAA Paper 2005-1265; 2005.

[33] Steger JL, Warming R. Flux vector splitting of the inviscid gas dynamic equations with application to finite difference methods. Journal of Computational Physics. 1981;40:263-93.

[34] Qiu J, Shu CW. On the Construction, Comparison, and Local Characteristic Decomposition for HighOrder Central WENO Schemes2002.

[35] Roe PL. Approximate Riemann Solvers, Parameter Vectors and Difference Schemes. Journal of Computational Physics. 1981;43:357-72.

[36] Lele SK. Compact Finite Difference Schemes with Spectral-like Resolution. Journal of Computational Physics. 1992;103:16-43.

[37] Visbal MR, Gaitonde DV. High-Order-Accurate Methods for Complex Unsteady Subsonic Flows. AIAA Journal. 1999;37:1231-9.

[38] Gottlieb S, Shu CW. Total variation diminishing Runge-Kutta schemes. Math Comput. 1998;67:73-85.

[39] Samtaney R, Pullin DI, Kosovic B. Direct numerical simulation of decaying compressible turbulence and shocklet statistics. Physics of Fluids. 2001;13:1415-30.

[40] Garnier E, Mossi M, Sagaut P, Comte P, Devilley M. On the Use of Shock-Capturing Schemes for Large-Eddy Simulation. Journal of Computational Physics. 1999;153:273-311.

[41] Jeong J, Hussain F. On the identification of a vortex. Journal of Fluid Mechanics. 1995;285:69-94.

[42] Kida S, Orszag SA. Energy and Spectral Dynamics in Forced Compressible Turbulence. Journal of Scientific Computing. 1990;5:85-125. 
[43] Moser RD, Kim J, Mansour NN. Direct numerical simulation of turbulent channel flow up to Re $\tau=590$. Physics of Fluids. 1999;11:943-5.

[44] Lenormand E, Sagaut P, Phuoc LT. Large eddy simulation of subsonic and supersonic channel flow at moderate Reynolds number. International Journal for Numerical Methods in Fluids. 2000;32:369-406.

[45] Sagaut P. Theoretical Background: Large-Eddy Simulation. In: Wagner C, Hüttl T, Sagaut P, editors. Large-Eddy Simulation for Acoustics: Cambridge: Cambridge University Press; 2007. p. 89-127.

[46] Eckelmann H. The structure of the viscous sublayer and the adjacent wall region in a turbulent channel flow. Journal of Fluid Mechanics. 1974;65:439-59.

[47] Niederschulte MA, Adrian RJ, Hanratty TJ. Measurements of turbulent flow in a channel at low Reynolds numbers. Experiments in Fluids. 1990;9,:222-30.

[48] Shutts WH, Hartwig WH, Weiler JE. Final report on turbulent boundary layer and skin friction measurements on a smooth, thermally insulated flat plate at supersonic speeds. Defense Research Laboratory; 1955.

[49] Wasistho B, Geurts BJ, Kuerten JGM. Simulation Techniques for Spatially Evolving InstabilitiesiIn Compressible Flow Over a Flat Plate. Computers \& Fluids. 1997;26:713-39.

[50] Rai MM, Moin P. Direct numerical simulation of transition and turbulence in a spatially evolving boundary layer. Journal of Computational Physics. 1993;109:169-92.

[51] Gao H, Fu D-X, Ma Y-W, Li X-L. Direct Numerical Simulation of Supersonic Turbulent Boundary Layer Flow. Chinese Physics Letters. 2005;22:1709-12.

[52] Pirozzoli S, Grasso F, Gatski TB. Direct numerical simulation and analysis of a spatially evolving supersonic turbulent boundary layer at $\mathrm{M}=2.25$. Physics of Fluids. 2004;16:530-45.

[53] Gloerfelt X, Lafon P. Direct Computation of the Noise induced by a turbulent flow through a diaphragm in a duct at low Mach number. Computer \& Fluids. 2008;37:388-401.

[54] Bookey P, Wyckham C, Smits A. New Experimental Data of STBLI at DNS/LES Accessible Reynolds Numbers. 43rd AIAA Aerospace Sciences Meeting and Exhibit. Reno, Nevada: AIAA Paper 2005-309; 2005. [55] Wu X, Moin P. Direct numerical simulation of turbulence in a nominally zero-pressure-gradient flatplate boundary layer. Journal of Fluid Mechanics. 2009;630:5-41.

[56] Dupont P, Haddad C, Debieve JF. Space and time organization in a shock-induced separated boundary layer. J Fluid Mech. 2006;559:255-77.

[57] Humble RA, Scarano F, van Oudheusden BW. Particle image velocimetry measurements of a shock wave/turbulent boundary layer interaction. Experiments In Fluids. 2007;43:173-83.

[58] Humble RA, Elsinga GE, Scarano F, van Oudheusden BW. Three-dimensional instantaneous structure of a shock wave turbulent boundary layer interaction. Journal of Fluid Mechanics. 2009;622:33-62.

[59] Purtell LP, Klebanoff PS, Buckley FT. Turbulent boundary layer at low Reynolds number. Physics of Fluids. 1981;24:802-11.

[60] Erm LP, Joubert PN. Low Reynolds number turbulent boundary layers. Journal of Fluid Mechanics. 1991;230:1-44.

[61] Spalart PR. Direct numerical simulation of a turbulent boundary layer up to $R e_{\theta}=1410$. Journal of Fluid Mechanics. 1988;187:61-98. 
[62] Pirozzoli S, Bernardini M, Grasso F. Direct numerical simulation of transonic shock/boundary layer interaction under conditions of incipient separation. Journal of Fluid Mechanics. 2010;657:361-93.

[63] Pirozzoli S, Grasso F. Direct numerical simulation of impinging shock wave/turbulent boundary layer interaction at $\mathrm{M}=2.25$. Physics of Fluids. 2006;18:065113.

[64] Priebe S, Wu M, Martin MP. Direct Numerical Simulation of a Reflected-Shock-Wave/TurbulentBoundary-Layer Interaction. AIAA Journal. 2009;47:1173-85.

[65] Zhou J, Adrian RJ, Balachandar S, Kendall TM. Mechanisms for generating coherent packets of hairpin vortices in channel flow. Journal of Fluid Mechanics. 1999;387:353-96.

[66] Adrian RJ, Meinhart CD, Tomkins CD. Vortex organization in the outer region of the turbulent boundary layer. Journal of Fluid Mechanics. 2000;422:1-54.

[67] Jammalamadaka A, Li Z, Jaberi F. Numerical Investigations of Shock Wave Interactions with a Supersonic Turbulent Boundary Layer. Physics of Fluids. 2014;26.

[68] Pirozzoli S, Bernardini M. Direct numerical simulation database for impinging shock wave/turbulent boundary-layer interaction. AIAA Journal. 2011;49:1307-12. 